JOURNAL OF THE

AMERICAN MATHEMATICAL SOCIETY

Volume 21, Number 1, January 2008, Pages 63-100

S 0894-0347(07)00559-0

Article electronically published on March 5, 2007

\title{
MACH CONFIGURATION IN PSEUDO-STATIONARY COMPRESSIBLE FLOW
}

\author{
SHUXING CHEN
}

\section{INTRODUCTION}

When a shock front in unsteady compressible flow is moving along an inclined ramp, the incident shock will be reflected by the ramp, as it moves forward. In the process of reflection, various reflected wave pattern may occur depending on the slope of the ramp and the strength of the incident shock 14, 23. This problem is one of the most important multidimensional prototype problems in gas dynamics. Generally, if the angle of inclination is larger than a critical value, then the reflected shock starting from the intersection of the incident shock with the ramp forms a smooth bubble, which expands when time goes on; such a reflection is called regular reflection. On the other hand, if the angle of inclination is small, then the intersection of the incident shock and the reflected shock will not touch the ramp, and an additional shock front called Mach stem appears, which connects the ramp with the intersection of the incident shock and the reflected shock. In this case three shock fronts meet at one point and form a triple shock configuration. An important fact is that the structure solely containing three shock fronts separating the neighborhood of the triple intersection into three zones with different continuous states does not exist [14, 27. Many physical experiments indicate that there is a slip line issuing from the triple intersection. Such a local wave pattern is called Mach configuration or Mach structure. Correspondingly, the whole process of reflection is called Mach reflection. Because of the complexity of the whole picture of Mach reflection, it is natural and necessary to start work on getting a clear understanding of the local Mach configuration.

Mach reflection also occurs in various other cases for compressible flow. For instance, in the study on oblique shock reflection in stationary compressible flow von Neumann indicated that (see [14, 25]) if the incident shock hit a wall with an angle larger than a critical value, then the reflection must be Mach reflection. Many physical experiments, approximate analysis and numerical computations verified the appearance of Mach configuration, and a tremendous amount of literature is devoted to study such problems (see [3, 4, 11, 19, 20, 24, and the references therein). However, for the rigorous proof on the stability of Mach configuration we can only mention a related result on stationary flow in [13].

Received by the editors November 14, 2005.

2000 Mathematics Subject Classification. Primary 35L65, 35L67, 76N10.

Key words and phrases. Euler system, Mach configuration, pseudo-stationary flow, free boundary problem, elliptic-hyperbolic composed system.

(C)2007 American Mathematical Society Reverts to public domain 28 years from publication 
The purpose of this paper is to give a mathematical confirmation for the Mach configuration appearing in the problem of shock reflection by ramp. Because of the spacial geometry of this problem one can use self-similar coordinates to simplify the analysis. In this coordinate system the incident shock, the reflected shock and the Mach stem become fixed curves, while the system for unsteady compressible flow becomes a new system called the pseudo-stationary Euler system [10. Correspondingly, the problem of verifying the Mach configuration (also for determining the whole wave pattern) can be described by solving a free boundary value problem of the pseudo-stationary Euler system. To achieve our purpose, we will give a precise mathematical formulation of such a free boundary value problem in Section 2, while the main result as well as the whole strategy of this paper, will be given in the end of Section 2.

\section{Preliminary preparation}

Consider the case when a plane shock with constant speed $w_{*}$ hits a straight ramp. Assume that a plane shock front which is perpendicular to a wall moves along the wall and hits a ramp with inclined angle $\theta_{*}$ at the time $t=0$. For $t>0$ the incident shock continuously moves forward, and a reflected shock front appears. As mentioned above, when $\theta_{*}$ is larger than a critical value depending on the parameters of the flow ahead and behind the shock front, then the reflected shock forms a bubble, which expands when $t$ increases. Such a reflection is called regular reflection and is discussed in [14, 26, 27]. Otherwise, when $\theta_{*}$ is smaller than the critical value, Mach reflection occurs. In this case three shock frontsincident, reflected and Mach stem - meet at one point and another additional slip line issues from the triple intersection as indicated by physical observation. In this paper we will not discuss much about the regular reflection. Instead, our attention is concentrated on confirming the reasonableness of the Mach configuration from a mathematical viewpoint. That is, we will give a rigorous proof on the local existence and stability of the solution with the above-mentioned wave pattern to the Euler system of compressible flow.

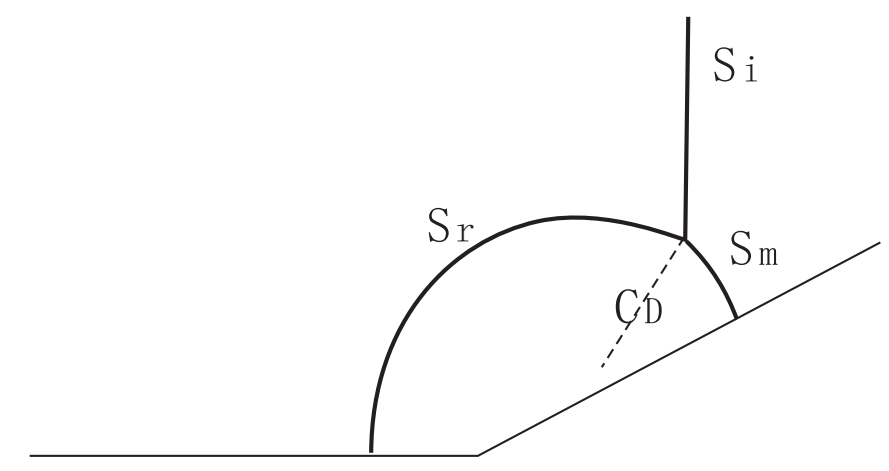

FIGURE 1. Mach reflection of shock by ramp 
The Euler system of unsteady compressible flow in two-dimensional space is

$$
\left\{\begin{array}{l}
\frac{\partial \rho}{\partial t}+\frac{\partial(\rho u)}{\partial x}+\frac{\partial(\rho v)}{\partial y}=0 \\
\frac{\partial}{\partial t}(\rho u)+\frac{\partial}{\partial x}\left(p+\rho u^{2}\right)+\frac{\partial}{\partial y}(\rho u v)=0 \\
\frac{\partial}{\partial t}(\rho v)+\frac{\partial}{\partial x}(\rho u v)+\frac{\partial}{\partial y}\left(p+\rho v^{2}\right)=0 \\
\frac{\partial}{\partial t}(\rho E)+\frac{\partial}{\partial x}(\rho u E+p u)+\frac{\partial}{\partial y}(\rho v E+p v)=0
\end{array}\right.
$$

where $E=\frac{1}{2}\left(u^{2}+v^{2}\right)+e$. The unknowns $(u, v), p, \rho, e$ stand for the velocity, pressure, density and inner energy of the flow, respectively. In the case of polytropic gas we have $p=A(s) \rho^{\gamma}$, where $s$ is entropy, $A(s)=(\gamma-1) \exp \left(c_{v}^{-1}\left(s-s_{*}\right)\right)$ with $c_{v}, s_{*}$ given constants. Also, $e+\frac{p}{\rho}=\frac{\gamma}{\gamma-1} \frac{p}{\rho}=\frac{a^{2}}{\gamma-1}$, where $a$ is the sonic speed of the flow. The system (2.1) can also be written in the matrix form

$$
\begin{aligned}
& \left(\begin{array}{cccc}
\rho & & & \\
& \rho & & \\
& a^{-2} \rho^{-1} & \\
& & & 1
\end{array}\right) \frac{\partial}{\partial t}\left(\begin{array}{l}
u \\
v \\
p \\
s
\end{array}\right)+\left(\begin{array}{cccc}
\rho u & & 1 & \\
& \rho u & & \\
1 & & a^{-2} \rho^{-1} u & \\
& & &
\end{array}\right) \frac{\partial}{\partial x}\left(\begin{array}{l}
u \\
v \\
p \\
s
\end{array}\right) \\
& +\left(\begin{array}{cccc}
\rho v & & & \\
& \rho v & 1 & \\
& 1 & a^{-2} \rho^{-1} v & \\
& & & v
\end{array}\right) \frac{\partial}{\partial y}\left(\begin{array}{l}
u \\
v \\
p \\
s
\end{array}\right)=0 .
\end{aligned}
$$

In the case when a shock front $y=\phi(t, x)$ (or a contact discontinuity as well) appears in the flow field, the Rankine-Hugoniot conditions for the parameters of the flow in both sides should be satisfied. That is

$$
\left[\begin{array}{c}
\rho \\
\rho u \\
\rho v \\
\rho E
\end{array}\right] \phi_{t}+\left[\begin{array}{c}
\rho u \\
p+\rho u^{2} \\
\rho u v \\
\rho u E+p u
\end{array}\right] \phi_{x}-\left[\begin{array}{c}
\rho v \\
\rho u v \\
p+\rho v^{2} \\
\rho u E+p v
\end{array}\right]=0 .
$$

Besides, the flow parameters on both sides of the shock front have to satisfy the entropy condition, which implies that the pressure or the density must increase when the flow passes across the shock front.

Due to the special geometry, the form of the whole problem will not be changed under the transformation $t \rightarrow \alpha t, x \rightarrow \alpha x, y \rightarrow \alpha y$, so we can only consider selfsimilar solutions in our problem. It means that all flow parameters in the equation and the boundary conditions are only the function of $\tilde{\xi}=\frac{x}{t}, \tilde{\eta}=\frac{y}{t}$. Correspondingly, the function $\phi(t, x)$ in the equation defining the shock front is homogeneous of degree 1 , so that the equation of the shock front can be written as $\tilde{\eta}=\psi(\tilde{\xi})$. Therefore, the system (2.1), its matrix form (2.2) and the Rankine-Hugoniot conditions 
(2.3) can be written as

$$
\begin{aligned}
& \left\{\begin{array}{l}
\frac{\partial(\rho(u-\tilde{\xi}))}{\partial \tilde{\xi}}+\frac{\partial(\rho(v-\tilde{\eta}))}{\partial \tilde{\eta}}+2 \rho=0, \\
\frac{\partial}{\partial \tilde{\xi}}(p+\rho u(u-\tilde{\xi}))+\frac{\partial}{\partial \tilde{\eta}}(\rho u(v-\tilde{\eta}))+2 \rho u=0, \\
\frac{\partial}{\partial \tilde{\xi}}(\rho v(u-\tilde{\xi}))+\frac{\partial}{\partial \tilde{\eta}}(p+\rho v(v-\tilde{\eta}))+2 \rho v=0, \\
\frac{\partial}{\partial \tilde{\xi}}(\rho(u-\tilde{\xi}) E+p u)+\frac{\partial}{\partial \tilde{\eta}}(\rho(v-\tilde{\eta}) E+p v)+2 \rho E=0,
\end{array}\right. \\
& \left(\begin{array}{cccc}
\rho(u-\tilde{\xi}) & & 1 & \\
1 & \rho(u-\tilde{\xi}) & a^{-2} \rho^{-1}(u-\tilde{\xi}) & \\
1 & & & u-\tilde{\xi}
\end{array}\right) \frac{\partial}{\partial \tilde{\xi}}\left(\begin{array}{l}
u \\
v \\
p \\
s
\end{array}\right) \\
& +\left(\begin{array}{cccc}
\rho(v-\tilde{\eta}) & & & \\
& \rho(v-\tilde{\eta}) & 1 & \\
& 1 & a^{-2} \rho^{-1}(v-\tilde{\eta}) & (v-\tilde{\eta})
\end{array}\right) \frac{\partial}{\partial \tilde{\eta}}\left(\begin{array}{l}
u \\
v \\
p \\
s
\end{array}\right)=0, \\
& {\left[\begin{array}{c}
\rho(u-\tilde{\xi}) \\
p+\rho u(u-\tilde{\xi}) \\
\rho v(u-\tilde{\xi}) \\
\rho E(u-\tilde{\xi})+p u
\end{array}\right] \psi_{\tilde{\xi}}-\left[\begin{array}{c}
\rho(v-\tilde{\eta}) \\
\rho u(v-\tilde{\eta}) \\
p+\rho v(v-\tilde{\eta}) \\
\rho E(v-\tilde{\eta})+p v
\end{array}\right]=0}
\end{aligned}
$$

respectively.

The system (2.4) or (2.5) describes the pseudo-stationary flow. If we introduce the pseudo-velocity $(U, V)$ with $U=u-\tilde{\xi}, V=v-\tilde{\eta}$, then the system (2.5) takes the form

$$
\begin{aligned}
& \left(\begin{array}{cccc}
\rho U & & 1 & \\
& \rho U & & \\
1 & & a^{-2} \rho^{-1} U & \\
& &
\end{array}\right) \frac{\partial}{\partial \tilde{\xi}}\left(\begin{array}{c}
U \\
V \\
p \\
s
\end{array}\right) \\
& +\left(\begin{array}{cccc}
\rho V & & & \\
& \rho V & 1 & \\
& 1 & a^{-2} \rho^{-1} V & \\
& & & V
\end{array}\right) \frac{\partial}{\partial \tilde{\eta}}\left(\begin{array}{c}
U \\
V \\
p \\
s
\end{array}\right)+\left(\begin{array}{c}
\rho U \\
\rho V \\
2 \\
0
\end{array}\right)=0 .
\end{aligned}
$$

Notice that if the pseudo-velocity $(U, V)$ is replaced by $(u, v)$, then the system (2.7) has the same principal part as that for the steady Euler system. That is why the flow described by (2.4) or (2.5) is called pseudo-stationary. Correspondingly, the Rankine-Hugoniot conditions take the same form as those in the steady case. In fact, the first equation in (2.6) is

$$
[\rho U] \psi_{\tilde{\xi}}-[\rho V]=0
$$

By using the first two equations, one has

$$
\begin{aligned}
& {\left[p+\rho U^{2}\right] \psi_{\tilde{\xi}}-[\rho U V]=\left[p+\rho(u-\tilde{\xi})^{2}\right] \psi_{\tilde{\xi}}-[\rho(u-\tilde{\xi})(v-\tilde{\eta})] } \\
= & {[p+\rho u(u-\tilde{\xi})] \psi_{\tilde{\xi}}-[\rho u(v-\tilde{\eta})]-\tilde{\xi}\left([\rho(u-\tilde{\xi})] \psi_{\tilde{\xi}}+[\rho(v-\tilde{\eta})]\right) . }
\end{aligned}
$$


Therefore, the second equation of (2.6) takes the form

$$
\left[p+\rho U^{2}\right] \psi_{\tilde{\xi}}-[\rho U V]=0 .
$$

Similarly, the third equation of (2.6) is

$$
[\rho U V] \psi_{\tilde{\xi}}-\left[p+\rho V^{2}\right]=0 .
$$

Finally, we have

$$
\begin{aligned}
{[\rho E(u-\tilde{\xi})+p u]=} & {\left[\rho e U+\frac{\rho}{2}\left(U^{2}+V^{2}\right) U+p U\right] } \\
& +\tilde{\xi}[p+\rho u U]+\tilde{\eta}[\rho v U]-\frac{\tilde{\xi}^{2}+\tilde{\eta}^{2}}{2}[\rho U]
\end{aligned}
$$

and similar expression for $[\rho E(v-\tilde{\eta})+p v]$. Then by using the first three equations in (2.6), we obtain

$$
[\rho \tilde{E} U+p U] \tilde{\psi}_{\tilde{\xi}}-[\rho \tilde{E} V+p V]=0,
$$

where $\tilde{E}=e+\frac{1}{2}\left(U^{2}+V^{2}\right)$. Obviously, the conditions (2.8)-(2.11) take the same form as the Rankine-Hugoniot conditions in steady flow.

Since the system (2.7) and the conditions (2.8)-(2.11) are similar to those for stationary compressible flow, then we can use the corresponding results in stationary flow to study the pseudo-stationary case. Particularly, the shock polar analysis can be applied to give a basic structure at the neighborhood of the singular point. Next we recall some basic facts for $(p, \theta)$ shock polar, which can be applied to analyze the structure of the wave pattern near the triple point.

The incident plane shock has velocity $w_{*}$ parallel to the $x$-axis; hence on the $(\tilde{\xi}, \tilde{\eta})$ plane it can be represented by a fixed line $\tilde{\xi}=\tilde{\xi}_{0}\left(=w_{*}\right)$, perpendicular to the $\tilde{\xi}$-axis. Assume that the triple intersection point is $O^{\prime}:\left(\tilde{\xi}_{0}, \tilde{\eta}_{0}\right)$. Then at $O^{\prime}$ the pseudo-velocity for the above four regions is given by $\left(U_{i}, V_{i}\right)=\left(u_{i}-\right.$ $\left.\tilde{\xi}_{0}, v_{i}-\tilde{\eta}_{0}\right)$, respectively. The direction of the shock front is determined by $\theta_{i}=$ $\arctan \left(V_{i} / U_{i}\right)$. Denote the state in the domain $\widetilde{O}$ ahead of the incident shock front $\mathbf{S}_{\mathbf{i}}$ by $\left(u_{0}, v_{0}, p_{0}, s_{0}\right)$ with $\left(u_{0}=0, v_{0}=0\right)$ and the state in the domain $\widetilde{I}$ behind the shock front $\mathbf{S}_{\mathbf{i}}$ by $\left(u_{1}, v_{1}, p_{1}, s_{1}\right)$ with $\left(u_{1}=q_{\infty}, v_{1}=0\right)$, which can be easily determined by $\left(u_{0}, v_{0}, p_{0}, s_{0}\right)$ and the Rankine-Hugoniot conditions on $\mathbf{S}_{\mathbf{i}}$. Denote the unknown state in the domain $\widetilde{I I}$ between the slip line $\mathbf{C}_{\mathbf{D}}$ and the reflected shock front $\mathbf{S}_{\mathbf{r}}$ by $\left(u_{2}, v_{2}, p_{2}, s_{2}\right)$ and the state in the domain $\widetilde{I I I}$ between the slip line and the Mach stem $\mathbf{S}_{\mathbf{m}}$ by $\left(u_{3}, v_{3}, p_{3}, s_{3}\right)$. Such a configuration near $O^{\prime}$ is called Mach configuration.

The shock polar analysis in stationary flow is often applied to determine the basic structure of the wave pattern involving shock fronts. Since the Rankine-Hugoniot condition for pseudo-stationary flow takes the same form as that for the stationary case, then the shock polar analysis can also be applied to the pseudo-stationary flow. Indeed, one can regard the pseudo-velocity $(U, V)$ for the pseudo-stationary flow as regular velocity $(u, v)$ for a stationary flow and then use shock polar analysis to determine the direction of the three shock fronts and the slip line at $O^{\prime}$. Assume that the pseudo-velocity $\left(U_{0}, V_{0}\right)$ is supersonic $\left(U_{0}^{2}+V_{0}^{2}>a_{0}^{2}\right)$. Then on $(p, \theta)$ plane one can draw a shock polar $\gamma_{0}$ with $P_{0}\left(p_{0}, \theta_{0}\right)$ as its double point (see Figure 2). Each point on $\gamma_{0}$ represents a state on the $(p, \theta)$ plane, which can be connected with $P_{0}$ via a shock front. Therefore, on the $(p, \theta)$ plane the state in the domain $\widetilde{I}$ can be represented by some point $P_{1}$ on the loop $\gamma_{0}$. If $P_{1}$ is not far from $P_{0}$, then the 


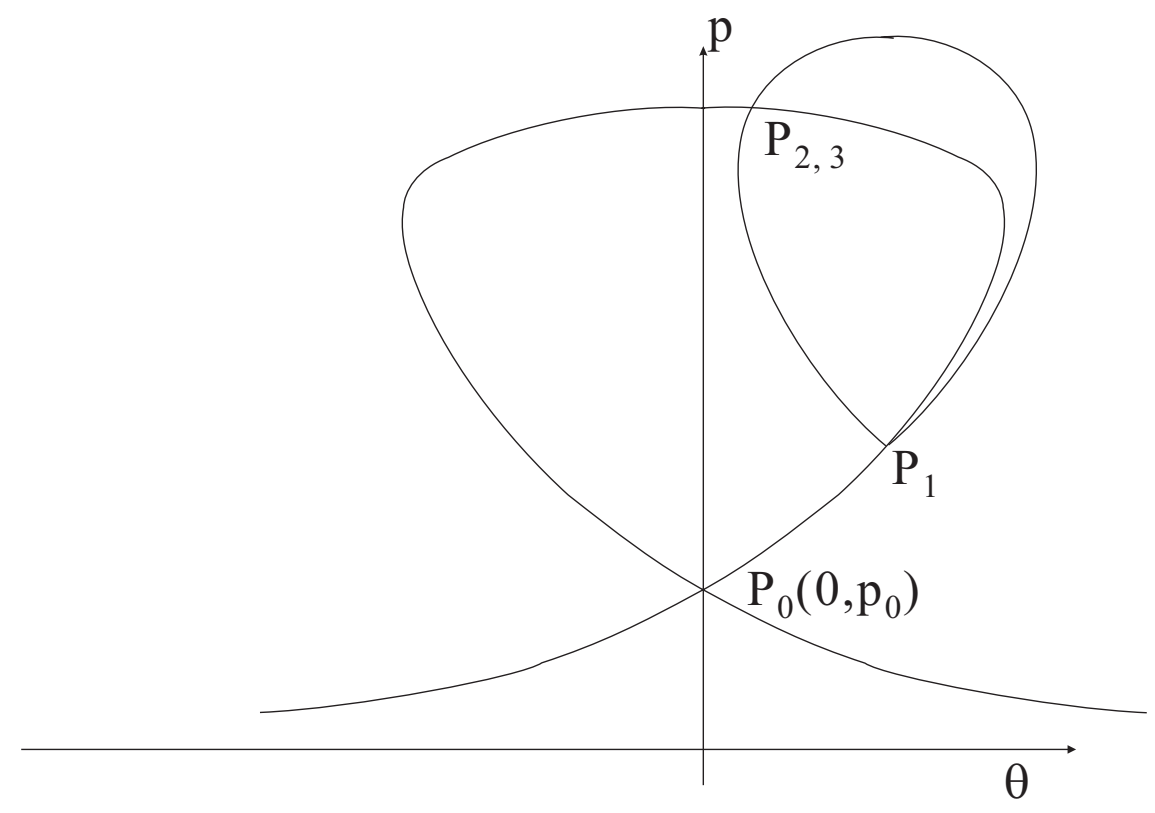

Figure 2. $(\theta, p)$-shock polar for Mach configuration

pseudo-velocity behind the incident shock front $\mathbf{S}_{\mathbf{i}}$ is still supersonic, so that one can draw another shock polar $\gamma_{1}$ with $P_{1}\left(p_{1}, \theta_{1}\right)$ as its double point. If the loop $\gamma_{1}$ intersects with the loop $\gamma_{0}$ at a point, then the point can be connected with $P_{0}$ via $\gamma_{0}$ and with $P_{1}$ via $\gamma_{1}$. To distinguish the two cases, we use two letters $P_{2}$ and $P_{3}$ to name the intersection, where $P_{2}$ corresponds to the state $\widetilde{\mathbf{U}}_{2}=\left(U_{2}, V_{2}, p_{2}, s_{2}\right)$, connecting with $\widetilde{\mathbf{U}}_{0}=\left(U_{0}, V_{0}, p_{0}, s_{0}\right)$ via Mach stem $\mathbf{S}_{\mathbf{m}}$, and $P_{3}$ corresponds to the state $\widetilde{\mathbf{U}}_{3}=\left(U_{3}, V_{3}, p_{3}, s_{3}\right)$, connecting with $\widetilde{\mathbf{U}}_{1}=\left(U_{1}, V_{1}, p_{1}, s_{1}\right)$ via the reflected shock front $\mathbf{S}_{\mathbf{r}}$. The fact that $P_{2}$ coincides with $P_{3}$ on the $(p, \theta)$ plane means that $p$ and $V / U(=\tan \theta)$ for the state $\widetilde{\mathbf{U}}_{2}$ and $\widetilde{\mathbf{U}}_{3}$ are equal. However, other flow parameters for these two states are not the same. Then on the $(\tilde{\xi}, \tilde{\eta})$ plane the states $\tilde{\mathbf{U}}_{2}$ and $\tilde{\mathbf{U}}_{3}$ must be separated by a contact discontinuity. Therefore, by adding an additional contact discontinuity, we obtain a wave pattern, which contains three straight shock fronts and a slip line. These four waves divide the neighborhood of $O^{\prime}$ into four regions as $\widehat{O}, \widehat{I}, \widehat{I I}, \widehat{I I I}$, which are the approximation of $\widetilde{O}, \widetilde{I}, \widetilde{I I}, \widetilde{I I I}$ locally.

The above analysis gives us four pseudo-constant states $\left(U_{j}, V_{j}, p_{j}, s_{j}\right)$ with $0 \leq j \leq 3$, which are separated by three straight shock fronts and one contact discontinuity. Since the coordinates of $O^{\prime}$ are $\left(\tilde{\xi}_{0}, \tilde{\eta}_{0}\right)$, then any straight line with slope $k$ issuing from $O^{\prime}$ satisfies

$$
\left(\tilde{\xi}-\tilde{\xi}_{0}, \tilde{\eta}-\tilde{\eta}_{0}\right) \cdot(k,-1)=0 .
$$

It turns out, when $\mathbf{S}$ is a straight shock front issuing from $O^{\prime}$ and the flow parameters $(u, v, p, s)$ on both sides are constants, then the satisfaction of Rankine-Hugoniot conditions (2.8) - 2.11) at $O^{\prime}$ also leads to the satisfaction of these conditions at any other point on the shock front on $\mathbf{S}$. Therefore, the four pseudo-constant states 
$\left(U_{j}, V_{j}, p_{j}, s_{j}\right)$ together with three straight shock fronts and one contact discontinuity determined by the shock polar analysis actually form a flat Mach configuration, which is denoted by $\left(\widetilde{\mathbf{U}}_{0}^{0}, \mathbf{S}_{\mathbf{i}}{ }^{0}, \widetilde{\mathbf{U}}_{1}^{0}, \mathbf{S}_{\mathbf{r}}{ }^{0}, \widetilde{\mathbf{U}}_{2}^{0}, \mathbf{C}_{\mathbf{D}}{ }^{0}, \widetilde{\mathbf{U}}_{3}^{0}, \mathbf{S}_{\mathbf{m}}{ }^{0}\right)$. This configuration is considered as a ground solution in this paper.

Generally, $\gamma_{1}$ and $\gamma_{0}$ have an intersection $P_{2}$ (or called $P_{3}$ ) other than $P_{1}$. In the Appendix we will prove the statement: if the parameters of the unperturbed upstream flow and the slope of the ramp satisfy the conditions

(1) $w_{*}>a_{0}$, which means that the relative velocity ahead of the shock front is supersonic,

(2) $\left(w_{*} \tan \theta_{*}\right)^{2}+\left(w_{*}-u_{1}\right)^{2}<a_{1}^{2}$, which means that the regular reflection at $\left(w_{*}, w_{*} \tan \theta_{*}\right)$ is impossible,

(3) the relative Mach number $M_{0}$ ahead of the incident shock is suitably large as required in Lemma 10.1

(4) $\theta_{P_{1}}>\theta_{\alpha}$, where $\theta_{\alpha}$ is a critical number defined in (10.17), then $\gamma_{1}$ and $\gamma_{0}$ must intersect at a point $P_{2}$ other than $P_{1}$, satisfying

$$
\theta_{P_{0}} \leq \theta_{P_{2}} \leq \theta_{P_{1}}
$$

The above conditions (1)-(4) are called Assumption (A) in the sequel.

For the global solution to Mach reflection, the flow in the domain $\widetilde{I I}$ and $\widetilde{I I I}$ should satisfy the impermeability boundary condition on the wall. However, the state $\widetilde{\mathbf{U}}_{2}$ (resp. $\widetilde{\mathbf{U}}_{3}$ ) behind the reflected shock (resp. Mach stem) for the flat Mach configuration obtained above does not satisfy the boundary condition on the wall generally, because the component of the velocity in the normal direction does not vanish. Therefore, in order to solve the problem on Mach reflection globally, we must consider the nonflat case. This means that the shock fronts are not straight, and the states behind the shock fronts are not piecewise constant anymore. Now that the downstream flow is not constant, we would also like to study the more general case, when the upstream flow is also not constant. It turns out, in order to study the global existence of Mach reflection, we first have to confirm the local stability of nonflat Mach configurations under perturbation at least locally, because any reasonable physical phenomena must be stable under perturbation.

Let us consider the following problem. Assume that the upstream slow in domain $\widetilde{O}$ is perturbed. We study the variation of the flow near $O^{\prime}$ in the regions $\widetilde{I}, \widetilde{I I}, \widetilde{I I I}$. First, since the pseudo-velocity of the flow in the domain $\widetilde{O}$ and $\widetilde{I}$ is supersonic, we can assume that the flow in these two regions is known due to the fact that the upstream part is not influenced by the downstream part in supersonic flow. Hence we only need to determine the state in the domain $\widetilde{I I}$ and $\widetilde{I I I}$, where the pseudo-velocity of the flow is subsonic.

The flow in domains $\widetilde{I I}$ and $\widetilde{I I I}$ must be determined globally, because the finite propagation property is not valid for subsonic flow. At this stage we are not quite clear on the situation of how the boundary conditions on the wall influence the solution. Therefore, to determine the flow in $\widetilde{I I}$ and $\widetilde{I I I}$, we add a cut-off boundary $L$ at the downstream part and impose a corresponding boundary condition on $L$. A natural boundary condition assigned on the cut-off boundary is that the pressure of the flow is given. Correspondingly, we are going to prove the wellposedness of the boundary value problem with boundary conditions produced by the perturbed upstream flow and the additional boundary conditions on the cut-off boundary. Such an approach has also been applied to deal with the problems on shock reflection 
for unsteady transonic small disturbance equation [9, 10] and on Mach reflection for the steady Euler system [13] .

Denote the unperturbed Mach configuration by $\left\{\widetilde{\mathbf{U}}_{0}^{0}, \mathbf{S}_{\mathbf{i}}^{\mathbf{0}}, \widetilde{\mathbf{U}}_{1}^{0}, \mathbf{S}_{\mathbf{r}}^{\mathbf{0}}, \widetilde{\mathbf{U}}_{2}^{0}, \mathbf{C}_{\mathbf{D}}^{\mathbf{0}}, \widetilde{\mathbf{U}}_{3}^{0}\right.$, $\left.\mathbf{S}_{\mathbf{m}}^{\mathbf{0}}\right\}$, and denote the perturbed Mach configuration by $\left\{\widetilde{\mathbf{U}}_{0}, \mathbf{S}_{\mathbf{i}}, \widetilde{\mathbf{U}}_{1}, \mathbf{S}_{\mathbf{r}}, \widetilde{\mathbf{U}}_{2}, \mathbf{C}_{\mathbf{D}}, \widetilde{\mathbf{U}}_{3}\right.$, $\left.\mathbf{S}_{\mathbf{m}}\right\}$, where $\left(\widetilde{\mathbf{U}}_{0}, \mathbf{S}_{\mathbf{i}}\right)$ is called upstream part, and the remainder of the configuration is called the downstream part. The above shock polar analysis shows that the state $\widetilde{\mathbf{U}}_{0}^{0}$ and the slope of the incident shock $\mathbf{S}_{\mathbf{i}}^{\mathbf{0}}$ determine the whole flat Mach configuration, provided the Assumption (A) is satisfied. It means that in the flat case the upstream part uniquely determines a Mach configuration. In this paper we will prove that a similar statement is also true for the nonflat case. The main results in this paper can be described by the following proposition:

Proposition 2.1. Assume a flat Mach configuration satisfies Assumption (A). If its upstream flow, the incident shock front and the pressure on the cut-off boundary are slightly perturbed, then the local structure of the Mach configuration still holds. Meanwhile, the downstream flow and the corresponding shock fronts and the slip line are also perturbed.

For our convenience in the following discussion we move the origin of the coordinate system to $O^{\prime}$ and then rotate the coordinate system around the origin $O^{\prime}$. In the sequel we denote the new coordinate variables by $(\xi, \eta)$ and let the $\xi$-axis be parallel to the tangential direction of the slip line. Under such translation and rotation the domains $\widetilde{O}, \widetilde{I}, \widetilde{I I}, \widetilde{I I I}$ are transformed to $\Omega_{0}, \Omega_{1}, \Omega_{2}, \Omega_{3}$, respectively; the incident shock $\mathbf{S}_{\mathbf{i}}$, the reflected shock $\mathbf{S}_{\mathbf{r}}$, the Mach stem $\mathbf{S}_{\mathbf{m}}$ are mapped to $S_{1}, S_{2}, S_{3}$, respectively, and the slip line is mapped to a curve $D$ tangential to the $\xi$-axis. Moreover, the state $\tilde{\mathbf{U}}_{i}^{0}$ becomes $\mathbf{U}_{i}^{0}$ by this translation and rotation. Notice that the system (2.7) is invariant under translation and rotation. Then the system and the Rankine-Hugoniot condition keep the same form in the new coordinates. Moreover, (10.7) confirmed in the Appendix means that in the $(\xi, \eta)$ coordinate system the $\eta$-component of the velocity is positive in $\Omega_{1}$ and negative in $\Omega_{0}$ near $O^{\prime}$. Then by using Rankine-Hugoniot conditions, we know near $O^{\prime}$ the Mach stem $S_{3}$ is located in the first quadrant, while the reflected shock front $S_{2}$ is located in the fourth quadrant, respectively. Since only the local stability of Mach configuration is studied in this paper, we take a small number $R$, such that in the circle $C_{R}$ with center $O^{\prime}$ and radius $R$ the whole shocks $S_{3}, S_{2}$ are located in the first and fourth quadrants, respectively (see Figure 3).

The equation of $S_{i}$ is denoted by $\eta=\psi_{i}(\xi)$ for $i=1,2,3$. The equation of the slip line $D$ is denoted by $\eta=\psi_{4}(\xi)$, which bears contact discontinuity and satisfies $\psi_{4}^{\prime}(0)=0$. The cut-off boundary in the downstream flow is given as $L: \xi=\ell$, where $\ell$ is a suitable small constant, because the discussion is local near $O^{\prime}$. Obviously, if $\ell$ is small, $L$ must intersect with $S_{2}$ and $S_{3}$, and the segment of $L$ between $S_{2}$ and $S_{3}$ is in $C_{R}$. Next we denote the domain enclosed by $L, D$ and $S_{i}$ by $\Omega_{i L}(i=2,3)$ and again use $\mathbf{U}=(U, V, p, s)$ to represent the parameters of the flow in the new coordinates system with a little abuse.

Let $\Omega_{0}^{e}$ be the domain on the $(\xi, \eta)$ plane satisfying

$$
(\xi, \eta) \in C_{R}, \quad \xi<\left\{\begin{array}{l}
\left(\psi_{3}^{0}\right)^{-1} \eta+e, \\
\left(\psi_{1}^{0}\right)^{-1} \eta+e,
\end{array}\right.
$$




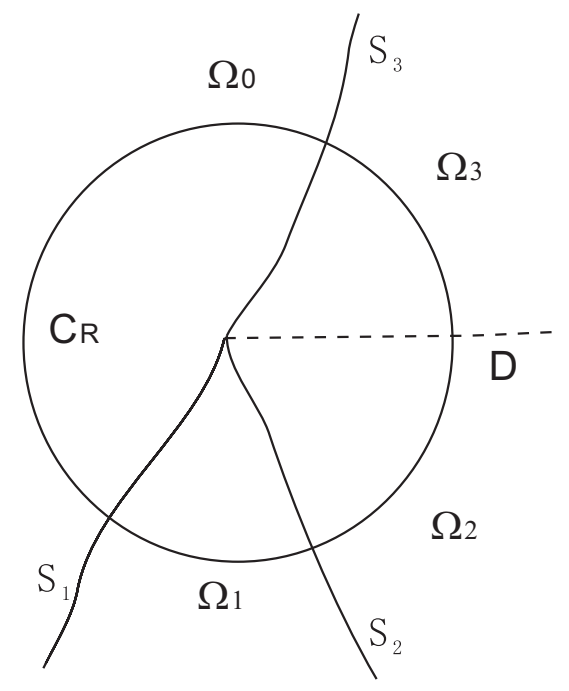

FiguRE 3. Flat Mach configuration in the $(\xi, \eta)$ coordinate system

where $e$ is a given small number. Let $N_{\varepsilon}$ be the neighborhood of $\mathbf{U}_{0}^{0}$

$$
N_{\varepsilon}=\left\{\mathbf{U}_{\mathbf{0}}(\xi, \eta) ; \mathbf{U}_{0}(0,0)=\mathbf{U}_{0}^{0},\left\|\mathbf{U}_{0}-\mathbf{U}_{0}^{0}\right\|_{\left.C^{2, \alpha_{0}\left(\Omega_{0}^{e}\right)}<\varepsilon\right\}}\right.
$$

let $b=\left(1+\psi_{0}^{2}\right)^{-1 / 2} R$, and let $L_{\varepsilon}$ be the neighborhood of $\mathbf{S}_{1}^{0}$

$$
L_{\varepsilon}=\left\{\psi_{1}(\xi) ; \psi_{1}(0)=0, \psi_{1}^{\prime}(0)=\psi_{1}^{0},\left\|\psi_{1}(\xi)-\psi_{1}^{0} \xi\right\|_{C^{2, \alpha_{0}(-b, 0)}}<\varepsilon\right\},
$$

where $\alpha_{0}$ is a number in $(0,1)$ determined later. Then Proposition 2.1 can be written as

Proposition 2.2. Assume that $\left(\mathbf{U}_{0}^{0}, S_{1}^{0}, \mathbf{U}_{1}^{0}, S_{2}^{0}, \mathbf{U}_{2}^{0}, D^{0}, \mathbf{U}_{3}^{0}, S_{3}^{0}\right)$ forms a flat Mach configuration satisfying Assumption (A). Assume that for given $R>0, e>0$ and small number $\varepsilon>0$, the upstream flow $\mathbf{U}_{\mathbf{0}} \in N_{\varepsilon}$ and $\psi_{1}(\xi) \in L_{\varepsilon}$. Then there is $R_{1} \in(0, R), \ell<R_{1}$ and a Mach configuration $\left\{\mathbf{U}_{0}, S_{1}, \mathbf{U}_{1}, S_{2}, \mathbf{U}_{2}, D, \mathbf{U}_{3}, S_{3}\right\}$ in $C_{R_{1}}$, satisfying the Euler system in domains, Rankine-Hugoniot conditions on the shock fronts and slip line, and the pressure control on the cut-off boundary. Moreover,

$$
\begin{gathered}
\psi_{i}(0)=0, \psi_{i}^{\prime}(0)=\psi_{i}^{0},\left\|\psi_{i}(\xi)-\psi_{i}^{0} \xi\right\|_{C^{2, \alpha}(0, \ell)}<\varepsilon_{1}, \quad i=2,3,4, \\
\mathbf{U}_{1}(0,0)=\mathbf{U}_{1}^{0},\left\|\mathbf{U}_{1}-\mathbf{U}_{1}^{0}\right\|_{C^{2, \alpha}\left(\Omega_{1} \cap C_{R_{1}}\right)}<\varepsilon_{1}, \\
\mathbf{U}_{i}(0,0)=\mathbf{U}_{i}^{0},\left\|\mathbf{U}_{i}-\mathbf{U}_{i}^{0}\right\|_{C^{1, \alpha}\left(\Omega_{i L}\right)}<\varepsilon_{1}, \quad i=2,3,
\end{gathered}
$$

where $\alpha \in\left(0, \alpha_{0}\right], \varepsilon_{1}$ tends to zero as $\varepsilon \rightarrow 0$.

Notice that for flat Mach configuration the flow $\mathbf{U}_{1}^{0}$ is also supersonic. Then its small perturbation is also supersonic. Since the theory of the quasilinear hyperbolic system in two variables (see 22]) indicates the existence of the flow $\mathbf{U}_{1}$, which is a perturbation of the flow $\mathbf{U}_{1}^{0}$, then to confirm Proposition 2.2, we only need to 
consider the following free boundary value problem:

$$
(F B):\left\{\begin{array}{l}
\text { the Euler system (2.7) in } \Omega_{2 L} \cup \Omega_{3 L}, \\
\text { the Rankine-Hugoniot conditions on } S_{2} \text { and } S_{3}, \\
p \text { and } V / U \text { are continuous on } D, \\
p=p_{2}^{0} \text { on } L .
\end{array}\right.
$$

In the problem $(F B)$ the data are given on the boundary $S_{2}, S_{3}$ and $L$. Similar to (2.13) we introduce

$$
\Omega_{1}^{e}=\left\{(\xi, \eta) \in C_{R} ;\left(\psi_{1}^{0}\right)^{-1} \eta<\xi<\left(\psi_{2}^{0}\right)^{-1} \eta+e\right\} .
$$

Then our task is reduced to proving the following theorem.

Theorem 2.1. Assume that $\left(\mathbf{U}_{0}^{0}, S_{1}^{0}, \mathbf{U}_{1}^{0}, S_{2}^{0}, \mathbf{U}_{2}^{0}, D, \mathbf{U}_{3}^{0}, S_{3}^{0}\right)$ forms a flat Mach configuration satisfying Assumption (A). Assume that for given $R>0, e>0$, small number $\varepsilon>0, \mathbf{U}_{i} \in C^{2, \alpha_{0}}\left(\Omega_{i}^{e}\right)(i=0,1)$ satisfies system (2.7) in $\Omega_{i}^{e}$ and the estimates

$$
\left\|\mathbf{U}_{i}-\mathbf{U}_{i}^{0}\right\|_{C^{2, \alpha}\left(\Omega_{i}^{e}\right)}<\varepsilon, \quad i=0,1 .
$$

Then one can find $\ell<R$ and $\psi_{i}(\xi)(i=2,3,4)$ defined in $(0, \ell)$ and $\mathbf{U}_{2}, \mathbf{U}_{3}$ defined in

$$
\Omega_{i L}=\left\{0<\xi<L,(-1)^{i} \psi_{i}(\xi)<(-1)^{i} \eta<(-1)^{i} \psi_{4}(\xi)\right\} \quad(i=2,3),
$$

respectively, such that the equations and boundary conditions in $(F B)$ are satisfied. Moreover,

$$
\begin{array}{r}
\left\|\psi_{i}(\xi)-\psi_{i}^{0} \xi\right\|_{C^{2, \alpha}(0, \ell)}<\varepsilon_{1}, \quad i=2,3,4, \\
\left\|\mathbf{U}_{i}-\mathbf{U}_{i}^{0}\right\|_{C^{1, \alpha}\left(\Omega_{i L}\right)}<\varepsilon_{1}, \quad i=2,3,
\end{array}
$$

where $\alpha \in\left(0, \alpha_{0}\right]$, $\varepsilon_{1}$ tends to zero as $\varepsilon \rightarrow 0$.

Remark. Since $p$ and $V / U$ are continuous on $D$, then by the property of Sobolev space we know that $p$ and $V / U$ belong to $H^{1}\left(\Omega_{2} \cup \Omega_{3}\right)$.

In order to prove Theorem 2.1, we should overcome some crucial difficulties. First, the problem is a free boundary value problem, in which the shock fronts $S_{2}$ and $S_{3}$ are unknown, and should be determined together with the solution of the problem. Second, the slip line $D$ is also an unknown curve located inside the flow field, so that the solution has discontinuity in the domain. Another difficulty is that the system (2.7), which is an elliptic-hyperbolic composed system, is neither hyperbolic nor elliptic. Finally, the corners of the domain also cause some trouble in our proof, because they weaken the smoothness of the precise solution and all approximate solutions for the elliptic equation near the corners.

Starting from the next section we will proceed with the proof of the main theorem. In Section 3 we introduce a generalized Lagrange transformation, which will straighten all stream lines, including the slip line. At this stage we are enlightened by the Lagrange transformation in one-dimensional unsteady flow (e.g. see [15, 28). In Section 4 we reduce the free boundary value problem into two problems. One is a fixed boundary value problem of the Euler system in a domain, whose shock boundary is temporarily fixed. The other is an initial value problem of ordinary differential equations updating the location of the shock fronts. Then the existence of the precise solution of the free boundary problem can be obtained by using the Schauder fixed point theorem (e.g. see [7, 8]). In Section 5 we decouple the Euler 
system (2.7), so that in the new form of the system the elliptic part and the hyperbolic part have been separated at the level of principal part. In Section 6 the whole problem is linearized based on this decoupled form. In Section 7 we concentrate our attention on establishing all necessary estimates for the linearized system. Then in order to overcome the trouble caused by the corners, we employ the corresponding results given by Grisvard and others in [5, 6, 16, 17. After these preparations we prove the convergence of approximate solutions of the linearized system in Section 8 and then finally prove Theorem 2.1 in Section 9. The conclusion obviously leads to the local existence of the Mach configuration in pseudo-stationary compressible flow. Finally, some explanations on Assumption (A) are given in the Appendix.

\section{Generalized Lagrange transformation}

In this section we will introduce a transformation $T$ to straighten all stream lines. In the process the slip line issuing from $O^{\prime}$ is transformed to the coordinate axis. The main idea of the transformation $T$ is to use the conservation law of mass to set up a new coordinate system moving with particles. This is similar to the Lagrange transformation in the study of one space dimensional conservation laws 28 and multidimensional stationary flow [12. Since in the system (2.4), $U=u-\tilde{\xi}, V=v-\tilde{\eta}$ are pseudo-velocity and since the equation of conservation of mass contains a zero-order term $2 \rho$, then we have to introduce an auxiliary integrating factor $\mu$ in the process of straightening all stream lines. This is the new ingredient different from the transformation employed in [28] and [13].

To simplify the notation, we will again call the new variables $x$ and $y$ (different from the variables employed in (2.1) $)$. Suppose that the solution $\mathbf{U}=(U, V, p, s)$ to the problem (FB) is known. We define $\mu$ by

$$
\left(U \frac{\partial}{\partial \xi}+V \frac{\partial}{\partial \eta}\right) \mu-2 \mu=0
$$

with the initial value $\mu=1$ on the shock front and then introduce variables $x, y$ by

$$
\left\{\begin{array}{l}
\frac{\partial x}{\partial \xi}=1, \quad \frac{\partial x}{\partial \eta}=0, \\
\frac{\partial y}{\partial \xi}=-\mu \rho V, \quad \frac{\partial y}{\partial \eta}=\mu \rho U, \\
x\left(\xi_{0}, \eta_{0}\right)=0, \quad y\left(\xi_{0}, \eta_{0}\right)=0 .
\end{array}\right.
$$

Since $\mu$ satisfies the equation (3.1), then the functions $x(\xi, \eta), y(\xi, \eta)$ are well defined. In fact, one can easily verify the equality

$$
\frac{\partial}{\partial \xi}(\mu \rho U)=-\frac{\partial}{\partial \eta}(\mu \rho V)
$$

by using (3.1) and the first equation in (2.4). Then we can define

$$
T: \quad x=\xi, \quad y=\int_{\left(\xi_{0}, \eta_{0}\right)}^{(\xi, \eta)}-\mu \rho V d \xi+\mu \rho U d \eta,
$$

which satisfy (3.2). From (3.2) we also have

$$
\left\{\begin{array}{l}
\frac{\partial \xi}{\partial x}=1, \quad \frac{\partial \xi}{\partial y}=0 \\
\frac{\partial \eta}{\partial x}=\frac{V}{U}, \quad \frac{\partial \eta}{\partial y}=\frac{1}{\mu \rho U}
\end{array}\right.
$$


and

$$
\begin{aligned}
& \frac{\partial}{\partial \xi}=\frac{\partial}{\partial x}-\mu \rho V \frac{\partial}{\partial y}, \frac{\partial}{\partial \eta}=\mu \rho U \frac{\partial}{\partial y}, \\
& U \frac{\partial}{\partial \xi}+V \frac{\partial}{\partial \eta}=U \frac{\partial}{\partial x} .
\end{aligned}
$$

The transform $T:(\xi, \eta) \mapsto(x, y)$ is called a generalized Lagrange transformation later. Under such a transformation the continuity equation becomes

$$
\frac{\partial}{\partial x}\left(\frac{1}{\mu \rho U}\right)=\frac{\partial}{\partial y}\left(\frac{V}{U}\right) .
$$

The two momentum equations can be reduced to

$$
\begin{gathered}
\rho U \frac{\partial U}{\partial x}+\frac{\partial p}{\partial x}-\mu \rho V \frac{\partial p}{\partial y}+\rho U=0, \\
\frac{1}{\mu} \frac{\partial V}{\partial x}+\frac{\partial p}{\partial y}+\frac{1}{\mu} \frac{V}{U}=0 .
\end{gathered}
$$

Direct computation also transforms the energy equation to

$$
\frac{\partial}{\partial x}\left(\tilde{E}+\frac{p}{\rho}\right)+\frac{\rho}{U}\left(U^{2}+V^{2}\right)=0 .
$$

Notice that

$$
\begin{gathered}
\frac{1}{\mu} \frac{\partial U}{\partial x}=\frac{\partial}{\partial x}\left(\frac{U}{\mu}\right)-U \frac{\partial}{\partial x}\left(\frac{1}{\mu}\right)=\frac{\partial}{\partial x}\left(\frac{U}{\mu}\right)+\frac{2}{\mu}, \\
\frac{1}{\mu} \frac{\partial V}{\partial x}=\frac{\partial}{\partial x}\left(\frac{V}{\mu}\right)+\frac{V}{U} \frac{2}{\mu} .
\end{gathered}
$$

Then the two momentum equations (3.7), (3.8) can be written as

$$
\begin{gathered}
\frac{\partial}{\partial x}\left(\frac{U}{\mu}+\frac{p}{\mu \rho U}\right)-\frac{\partial}{\partial y}\left(\frac{p V}{U}\right)+\frac{3}{\mu}=0, \\
\frac{\partial}{\partial x}\left(\frac{V}{\mu}\right)+\frac{\partial p}{\partial y}+\frac{3}{\mu} \frac{V}{U}=0
\end{gathered}
$$

with the form of conservation laws. Besides, the equation (3.1) can be written as

$$
U \frac{\partial}{\partial x} \mu-2 \mu=0 .
$$

The Rankine-Hugoniot conditions on the image $y=\chi(x)$ of shock front $\eta=\psi(\xi)$ are

$$
\left\{\begin{array}{l}
{\left[\frac{1}{\rho U}\right] \frac{\chi^{\prime}}{\mu}=-\left[\frac{V}{U}\right],} \\
{\left[\frac{1}{\rho U}\left(p+\rho U^{2}\right)\right] \frac{\chi^{\prime}}{\mu}=-\left[\frac{p V}{U}\right],} \\
{[V] \frac{\chi^{\prime}}{\mu}=[p],} \\
{\left[\tilde{E}+\frac{p}{\rho}\right]=0 .}
\end{array}\right.
$$

These equations can be directly derived from the equations (3.6), (3.9), (3.10), (3.11) with the corresponding form of conservation laws and can also be derived 
from (2.6) via

$$
\psi^{\prime}=\frac{\partial \eta}{\partial x}+\frac{\partial \eta}{\partial y} \chi^{\prime}=\frac{V}{U}+\frac{1}{\mu \rho U} \chi^{\prime} .
$$

Eliminating $\chi^{\prime}$ in (3.13), we have

$$
\left\{\begin{array}{l}
{[p]\left[U+\frac{p}{\rho U}\right]=-[V]\left[\frac{p V}{U}\right],} \\
{[p]\left[\frac{1}{\rho U}\right]=-[V]\left[\frac{V}{U}\right]} \\
{\left[e+\frac{1}{2}\left(U^{2}+V^{2}\right)+\frac{p}{\rho}\right]=0 .}
\end{array}\right.
$$

Denote the image of the shock front $S_{2}, S_{3}$ by $\Gamma_{2}, \Gamma_{3}$, the image of $D, L$ by $\Gamma_{D}, \Gamma_{L}$, and denote the image of $\Omega_{2 L}, \Omega_{3 L}$ by $\omega_{2}, \omega_{3}$. Then we are led to a new free boundary value problem on the $(x, y)$ plane as

$$
(F B)_{1}:\left\{\begin{array}{l}
\text { the Euler system }(\underline{3.6}),(\sqrt{3.9}),(3.10),(3.11) \text { with }(3.12) \text { in } \omega_{2} \cup \omega_{3}, \\
\text { Rankine-Hugoniot conditions }(3.13) \text { and } \mu=1 \text { on } \Gamma_{2} \text { and } \Gamma_{3}, \\
p \text { and } V / U \text { are continuous on } \Gamma_{D}, \\
p=p_{0} \quad \text { on } \Gamma_{L} .
\end{array}\right.
$$

\section{REDUCTION TO THE FIXED BOUNDARY VALUe PROBLEM}

To solve the problem $(F B)_{1}$, we reduce the whole free boundary value problem to two problems: one is a regular boundary value problem for the Euler system in a fixed domain, and the other is an initial value problem of ordinary differential equation updating the shock fronts. By using the solutions of these two problems, we establish a map defined on a closed convex set in a suitable functional space. Then the demonstration of the existence of the problem $(F B)_{1}$ amounts to proving the existence of a fixed point for such a given map.

In order to describe the process of the reduction, we introduce a set $K_{\zeta}$ as follows:

$$
\begin{array}{r}
K_{\zeta}=\left\{\left(\chi_{2}(x), \chi_{3}(x)\right) \in C^{2, \alpha}(0, \ell) ; \chi_{i}(0)=0, \chi_{i}^{\prime}(0)=\chi_{i}^{0},\right. \\
\left.\left\|\chi_{i}(x)\right\|_{C^{2, \alpha}(0, \ell)} \leq \zeta ; i=2,3\right\},
\end{array}
$$

where $\chi_{i}^{0}=\left(-\left[\frac{V}{U}\right] /\left[\frac{1}{\rho U}\right]\right)(0,0)$ is the slope of the shock front $\Gamma_{i}^{0}$ in the flat case. For any pair of functions $\left(\chi_{2}, \chi_{3}\right) \in K_{\zeta}$ we denote $\omega_{2}=\left\{0>y>\chi_{2}(x), 0<x<\right.$ $\ell\}, \omega_{3}=\left\{0<y<\chi_{3}(x), 0<x<\ell\right\}, \omega=\left\{\chi_{2}(x)<y<\chi_{3}(x), 0<x<\ell\right\}$, and we can define a boundary value problem on $\omega$.

$$
(N L):\left\{\begin{array}{l}
\text { the Euler system }(3.6),(3.9),(3.10),(3.11) \text { and }(3.12) \text { in } \omega \\
G^{a} \triangleq[p]\left[\frac{1}{\rho U}\right]+[W U][W]=0 \text { on } \Gamma_{2} \text { and } \Gamma_{3}, \\
G^{b} \triangleq[p]\left[U+\frac{p}{\rho U}\right]+[W U][p W]=0 \text { on } \Gamma_{2} \text { and } \Gamma_{3}, \\
G^{c} \triangleq\left[\tilde{E}+\frac{p}{\rho}\right]=0 \text { on } \Gamma_{2} \text { and } \Gamma_{3}, \\
\mu=1 \quad \text { on } \Gamma_{2} \text { and } \Gamma_{3}, \\
p \text { and } V / U \text { are continuous on } \Gamma_{D}, \\
p=p_{0} \text { on } \Gamma_{L}
\end{array}\right.
$$


where $W=V / U$. In (4.2) the boundary conditions are set as form (3.14), while one condition in the Rankine-Hugoniot conditions (3.13) is left to modify the position of the shock fronts. Since $\mu=1$ on $\Gamma_{2}$ and $\Gamma_{3}$, then $\mu$ does not appear in other boundary conditions on $\Gamma_{2}$ and $\Gamma_{3}$.

Again use $\mathbf{U}$ to denote the solution $(U, V, p, s, \mu)$ of the problem $(N L)$. When a solution $\mathbf{U}$ of the problem $(N L)$ is obtained, we set an initial value problem to modify the shock fronts. That is,

$$
\left\{\begin{array}{l}
\frac{d \Xi_{i}}{d x}=-\frac{\left[\frac{V}{U}\right]}{\left[\frac{1}{\rho U}\right]} \text { on } \Gamma_{i}, \quad i=2,3 \\
\Xi_{i}(0)=0
\end{array}\right.
$$

Since $\theta\left(P_{2,3}\right)$ satisfies $\theta\left(P_{0}\right)<\theta\left(P_{2,3}\right)<\theta\left(P_{1}\right)$ as shown in Section 2, then the angle between the tangential of $D$ with $S_{2}$ or $S_{3}$ is less than $\frac{\pi}{2}$. Hence the denominator $\left[\frac{1}{\rho U}\right]$ of (4.3) does not vanish near $O^{\prime}$, and the problem (4.3) is well defined. $\left(\chi_{2}(x), \chi_{3}(x)\right) \mapsto\left(\Xi_{2}(x), \Xi_{3}(x)\right)$ defines a map $\pi$ from $K_{\zeta}$ to $K_{\zeta}$. Our next step is to establish some estimates of the solution to (4.2) and (4.3), which ensure the compactness of the map $\pi$ from $K_{\zeta}$ to $K_{\zeta}$ for small $\zeta$. Since $K_{\zeta}$ is a closed convex set, then Schauder fixed point theorem will give a fixed point of $\pi$, which leads to a solution of the problem $(F B)_{1}$.

\section{Decoupling}

The Euler system in (4.2), as well as in (3.14), is hyperbolic if $U^{2}+V^{2}>a^{2}$ and is neither pure hyperbolic nor elliptic if $U^{2}+V^{2}<a^{2}$. In the domain $\omega_{2,3}$ the pseudo-velocity is subsonic, so the system in (4.2) is an elliptic-hyperbolic composed system. To deal with such a composed system, we reduce the system to a canonical form, which decouples the elliptic part and the hyperbolic part of the system at the level of the principal part (see [12, 13]). Write the system (4.2) in matrix form as

$$
\mathbf{A} \frac{\partial}{\partial x} \mathbf{U}+\mathbf{B} \frac{\partial}{\partial y} \mathbf{U}+\mathbf{D}=0
$$

where

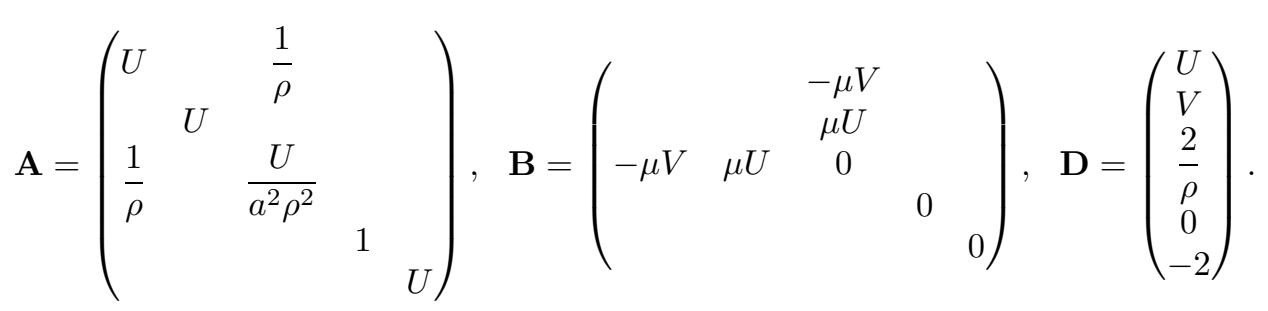


The characteristic polynomial of this system is

$$
\begin{aligned}
D(\lambda) & =\operatorname{det}(\lambda A-B) \\
& =\operatorname{det}\left[\begin{array}{cccc}
\lambda U & \lambda U & \frac{\lambda}{\rho}+\mu V & \\
\frac{\lambda}{\rho}+\mu V & -\mu U & \frac{\lambda U}{a^{2} \rho^{2}} & \\
& =\lambda^{3} U\left(\frac{\lambda^{2} U^{2}}{a^{2} \rho^{2}}-\left(\mu V+\frac{\lambda}{\rho}\right)^{2}-\mu^{2} U^{2}\right) .
\end{array}\right]
\end{aligned}
$$

$D(\lambda)=0$ has a triple root $\lambda=0$. Its other two roots satisfy

$$
\lambda^{2}\left(U^{2}-a^{2}\right)-2 \lambda a^{2} \rho \mu V-a^{2} \rho^{2} \mu^{2}\left(U^{2}+V^{2}\right)=0 .
$$

Hence

$$
\begin{gathered}
\lambda_{ \pm}=\frac{\mu a^{2} \rho V \pm \sqrt{a^{4} \rho^{2} \mu^{2} V^{2}+\mu^{2} a^{2} \rho^{2}\left(U^{2}+V^{2}\right)\left(U^{2}-a^{2}\right)}}{U^{2}-a^{2}} \\
=\frac{\mu a^{2} \rho V \pm \mu a \rho U \sqrt{U^{2}+V^{2}-a^{2}}}{U^{2}-a^{2}}
\end{gathered}
$$

Therefore, in the pseudo-supersonic region the equation $D(\lambda)=0$ has five real roots, and the system (4.2) is purely hyperbolic. However, in the pseudo-subsonic region the equation $D(\lambda)=0$ has three real roots and two complex roots, so that the system (4.2) is an elliptic-hyperbolic composed system.

Denote $\lambda_{ \pm}=\lambda_{R} \pm i \lambda_{I}$ in the pseudo-subsonic region, where

$$
\lambda_{R}=\frac{\mu a^{2} \rho V}{U^{2}-a^{2}}, \quad \lambda_{I}=\frac{\mu a \rho U \sqrt{a^{2}-U^{2}-V^{2}}}{U^{2}-a^{2}} .
$$

The left eigenvector of the matrix $\lambda_{ \pm} A-B$ is

$$
\ell_{ \pm}=\left(\frac{1}{U}\left(-\frac{\lambda_{ \pm}}{\rho}-\mu V\right), \mu, \lambda_{ \pm}, 0,0\right)
$$

For $\lambda_{ \pm}=\lambda_{R} \pm i \lambda_{I}$ we separate the real part and imaginary part of its eigenvector to obtain

$$
\begin{gathered}
\ell_{R}=\left(\frac{1}{U}\left(-\frac{\lambda_{R}}{\rho}-\mu V\right), \mu, \lambda_{R}, 0,0\right), \\
\ell_{I}=\left(-\frac{1}{\rho U} \lambda_{I}, 0, \lambda_{I}, 0,0\right) .
\end{gathered}
$$

Besides, the left eigenvectors of $\lambda A-B$ corresponding to the eigenvalue 0 is $\ell_{3}=$ $(U, V, 0,0,0), \ell_{4}=(0,0,0,1,0)$ and $\ell_{5}=(0,0,0,0,1)$. Multiplying the system by these left eigenvectors, we obtain the canonical form of the system. For instance, multiplying the system by $\ell_{ \pm}$leads to

$$
\ell_{ \pm} \mathbf{A}\left(\frac{\partial}{\partial \xi}+\lambda_{ \pm} \frac{\partial}{\partial \eta}\right) \mathbf{U} \pm \ell_{ \pm} \mathbf{D}=0
$$


Separating its real part and imaginary part yields

$$
\begin{aligned}
& -V D_{R} U+U D_{R} V-\frac{\mu}{a \rho} \sqrt{a^{2}-U^{2}-V^{2}} D_{I} p+\frac{\lambda_{R}}{\rho}=0, \\
& -V D_{I} U+U D_{I} V+\frac{\mu}{a \rho} \sqrt{a^{2}-U^{2}-V^{2}} D_{R} p+\frac{\lambda_{I}}{\rho}=0,
\end{aligned}
$$

where $D_{R}=\frac{\partial}{\partial x}+\lambda_{R} \frac{\partial}{\partial y}, D_{I}=\lambda_{I} \frac{\partial}{\partial y}$. Dividing (15.3), (5.4) by $U^{2}$ and setting $W=\frac{V}{U}$, we have

$$
\left\{\begin{array}{l}
D_{R} W-e D_{I} p+\frac{\lambda_{R}}{\rho U^{2}}=0 \\
D_{I} W+e D_{R} p+\frac{\lambda_{I}}{\rho U^{2}}=0
\end{array}\right.
$$

where $e=\frac{\mu}{a \rho U^{2}} \sqrt{a^{2}-U^{2}-V^{2}}$. Correspondingly, by multiplying the system (4.2) by $\ell_{3}, \ell_{4}$ and $\ell_{5}$ gives us three transport equations:

$$
\begin{gathered}
U \frac{\partial U}{\partial x}+V \frac{\partial V}{\partial x}+\frac{1}{\rho} \frac{\partial p}{\partial x}+\frac{U^{2}+V^{2}}{U}=0 \\
\frac{\partial s}{\partial x}=0 \\
U \frac{\partial \mu}{\partial x}-2 \mu=0
\end{gathered}
$$

For the polytropic gas, the equation (5.7) can be replaced by

$$
\frac{\partial p}{\partial x}-\frac{\gamma p}{\rho} \frac{\partial \rho}{\partial x}=0
$$

Now the system (5.5)-(5.8) has been decoupled at its principle level: (5.5) is an elliptic system, while the transport equations (5.6)-(5.8) are hyperbolic. In the sequel we will take $(U, W, p, \rho, \mu)$ as unknown functions instead of $(U, V, p, s, \mu)$ and still use the notation $\mathbf{U}$ to stand for all unknown functions. Therefore, the differential system in the problem $(N L)$ can be replaced by

$$
\left\{\begin{array}{l}
D_{R} W-e D_{I} p+\frac{\lambda_{R}}{\rho U^{2}}=0 \\
D_{I} W+e D_{R} p+\frac{\lambda_{I}}{\rho U^{2}}=0 \\
\frac{\partial U}{\partial x}+d_{W} \frac{\partial W}{\partial x}+d_{p} \frac{\partial p}{\partial x}+1=0 \\
\frac{\partial p}{\partial x}-\frac{\gamma p}{\rho} \frac{\partial \rho}{\partial x}=0 \\
U \frac{\partial \mu}{\partial x}-2 \mu=0
\end{array}\right.
$$

where $d_{W}=\frac{U^{2} W}{U+U W^{2}}, d_{p}=\frac{1}{\rho\left(U+U W^{2}\right)}$. 


\section{LINEARIZED PROBLEM}

The nonlinear fixed boundary value problem $(N L)$ will be solved by an iterative scheme. To this end we first have to linearize the problem. For given fixed domain $\omega=\omega_{2} \cup \omega_{3}$ and small number $\delta>0$, we define

$$
\begin{aligned}
\Sigma_{\delta}=\left\{\mathbf{U} \in L^{2}(\omega) ; p, W, \mu \in H^{1}(\omega), \mathbf{U}_{i}=\left.\mathbf{U}\right|_{\omega_{i}} \in C^{1, \alpha},\right. \\
\left.\mathbf{U}_{i}(0,0)=\mathbf{U}_{i}^{0}(i=2,3), \sum_{i=2,3}\left\|\mathbf{U}_{i}-\mathbf{U}_{i}^{0}\right\|_{C^{1, \alpha}\left(\bar{\omega}_{i}\right)} \leq \delta\right\} .
\end{aligned}
$$

Denote $H_{1}=\lambda_{R} /\left(\rho U^{2}\right), H_{2}=\lambda_{I} /\left(\rho U^{2}\right)$. For any $\mathbf{U} \in \Sigma_{\delta}$, we can linearize the system (5.10) to obtain

$$
\left\{\begin{array}{l}
D_{R} \delta W-e D_{I} \delta p+H_{1 \mathbf{U}} \delta \mathbf{U}=f_{1} \\
D_{I} \delta W+e D_{R} \delta p+H_{2 \mathbf{U}} \delta \mathbf{U}=f_{2} \\
\frac{\partial \delta U}{\partial x}+d_{w} \frac{\partial \delta W}{\partial x}+d_{p} \frac{\partial \delta p}{\partial x}=f_{3} \\
\frac{\partial \delta p}{\partial x}-\frac{\gamma p}{\rho} \frac{\partial \delta \rho}{\delta x}=f_{4} \\
U \frac{\partial \delta \mu}{\partial x}-2 \delta \mu=f_{5}
\end{array}\right.
$$

where $H_{i \mathbf{U}}$ is the Frechet derivative of $H_{i}$ with respect to $\mathbf{U}$. The linearization of the nonlinear boundary conditions $G^{a}=0, G^{b}=0, G^{c}=0$ on $\Gamma_{2,3}$ are

$$
\left\{\begin{array}{l}
\alpha^{a} \delta U+\beta^{a} \delta W+\gamma^{a} \delta p+\theta^{a} \delta \rho=g^{a}, \\
\alpha^{b} \delta U+\beta^{b} \delta W+\gamma^{b} \delta p+\theta^{b} \delta \rho=g^{b} \\
\alpha^{c} \delta U+\beta^{c} \delta W+\gamma^{c} \delta p+\theta^{c} \delta \rho=g^{c}
\end{array}\right.
$$

where $\alpha^{\sharp}, \beta^{\sharp}, \gamma^{\sharp}, \theta^{\sharp}$ are components of $G_{\mathbf{U}}^{\sharp}$ - the Frechet derivatives of $G^{\sharp}(\sharp=$ $a, b, c)$. Their expressions are

$$
\begin{gathered}
\alpha^{a}=-[p] \frac{1}{\rho U^{2}}+[W] W, \quad \beta^{a}=[W U]+[W] U, \\
\gamma^{a}=\left[\frac{1}{\rho U}\right], \quad \theta^{a}=-[p] \frac{1}{\rho^{2} U}, \\
\alpha^{b}=[p]\left(1-\frac{p}{\rho U^{2}}\right)+[p W] W, \quad \beta^{b}=[W U] p+U[p W], \\
\gamma^{b}=\left[U+\frac{p}{\rho U}\right]+[p]\left(\frac{1}{\rho U}\right)+[W U] W, \quad \theta^{b}=-[p] \frac{p}{\rho^{2} U}, \\
\alpha^{c}=U+W^{2} U, \quad \beta^{c}=W U^{2}, \\
\gamma^{c}=\frac{\gamma}{\gamma-1} \frac{1}{\rho}, \quad \theta^{c}=-\frac{\gamma}{\gamma-1} \frac{p}{\rho^{2}} .
\end{gathered}
$$

Lemma 6.1. Looking at (6.3) as a linear algebraic system for $\delta U, \delta W, \delta p, \delta \rho$, the variables $\delta U, \delta W, \delta \rho$ can be solved as functions of $\delta p$. That is, (6.3) can be rewritten as

$$
\left\{\begin{array}{l}
\delta W+\tau_{W} \delta p=g_{W} \\
\delta U+\tau_{U} \delta p=g_{U} \\
\delta \rho+\tau_{\rho} \delta p=g_{\rho}
\end{array}\right.
$$


Proof. It is enough to verify that the coefficient matrix

$$
\mathbf{M}=\left(\begin{array}{lll}
\alpha^{a} & \beta^{a} & \theta^{a} \\
\alpha^{b} & \beta^{b} & \theta^{b} \\
\alpha^{c} & \beta^{c} & \theta^{c}
\end{array}\right)
$$

is nonsingular. In fact, by continuity we only need to verify the fact for $W=0$, because $W$ is small in our whole discussion. In this case the matrix

$$
\begin{aligned}
\mathbf{M} & \sim\left(\begin{array}{ccc}
-\frac{[p]}{\rho U^{2}} & {[W U]+[W] U} & -[p] \frac{1}{\rho^{2} U} \\
{[p]\left(1-\frac{p}{\rho U^{2}}\right)} & {[W U] p+U[p W]} & -[p] \frac{p}{\rho^{2} U} \\
U & 0 & -\frac{\gamma}{\gamma-1} \frac{p}{\rho^{2}}
\end{array}\right) \\
& \sim\left(\begin{array}{ccc}
-\frac{[p]}{\rho U^{2}} & {[W U]+[W] U} & -[p] \frac{1}{\rho^{2} U} \\
{[p]} & U[p W]-[W] U p & 0 \\
U & 0 & -\frac{\gamma}{\gamma-1} \frac{p}{\rho^{2}}
\end{array}\right) \\
& \sim\left(\begin{array}{ccc}
-\frac{[p]}{\rho U^{2}} & -W_{-}\left(U+U_{-}\right) & -[p] \frac{1}{\rho^{2} U} \\
{[p]} & {[p] U W_{-}} & 0 \\
U & 0 & -\frac{\gamma}{\gamma-1} \frac{p}{\rho^{2}}
\end{array}\right) \\
& \sim\left(\begin{array}{ccc}
-\frac{[p]}{\rho U^{2}} & -\left(U+U_{-}\right) & -[p] \\
1 & U & 0 \\
1 & 0 & -\frac{\gamma}{\gamma-1} p
\end{array}\right) .
\end{aligned}
$$

Denoting by $\tau$ the angle of the normal direction of the shock front with $x$-axis, and denoting by $U_{n}, U_{t}$ the component of the pseudo-velocity $(U, V)$ in the normal and tangential directions of the shock front, respectively, we have

$$
\begin{gathered}
\rho U_{n}=\rho_{-} U_{n-}, p+\rho U_{n}^{2}=p_{-}+\rho_{-} U_{n-}^{2}, U_{t}=U_{t-}, \\
U=U_{n} \cos \tau+U_{t} \sin \tau, U_{-}=U_{n-} \cos \tau+U_{t} \sin \tau, \\
V=U_{t} \cos \tau-U_{n} \sin \tau, V_{-}=U_{t} \cos \tau-U_{n-} \ell \sin \tau .
\end{gathered}
$$

Therefore,

$$
\begin{aligned}
p & -p_{-}+\rho U^{2}-\rho U U_{-}=p-p_{-}+\rho\left(U_{n} \cos \tau+U_{t} \sin \tau\right)\left(U_{n}-U_{n-}\right) \cos \tau \\
& =-\rho U_{n}\left(U_{n}-U_{n-}\right)+\rho\left(U_{n}-U_{n-}\right)\left(U_{n} \cos ^{2} \tau+U_{t} \cos \tau \sin \tau\right) \\
& =\rho\left(U_{n}-U_{n-}\right)\left(-U_{n} \sin ^{2} \tau+U_{t} \cos \tau \sin \tau\right) \\
& =\rho\left(U_{n}-U_{n-}\right) V \sin \tau=0 .
\end{aligned}
$$


Hence the determinant of the last matrix in (6.5) is

$$
\begin{aligned}
\operatorname{det} & =\left|\begin{array}{ccc}
-\frac{\rho U\left(U-U_{-}\right)}{\rho U^{2}} & -\left(U+U_{-}\right) & -[p] \\
1 & U & 0 \\
1 & 0 & -\frac{\gamma}{\gamma-1} p
\end{array}\right| \\
& =-\frac{\gamma}{\gamma-1} p \cdot 2 U+[p] U \\
& =U\left(-\frac{\gamma+1}{\gamma-1} p-p_{-}\right)<0 .
\end{aligned}
$$

This means that the matrix $\mathbf{M}$ is nonsingular, so that we can find $\tau_{W}, \tau_{U}, \tau_{\rho}$ such that (6.4) holds.

The linearized form of the compatibility condition on $\Gamma_{D}$ is simply the continuity of $\delta p$ and $\delta W$, and the corresponding linearized boundary condition on $\Gamma_{L}$ is $\delta p=0$. Therefore, we obtain a linearized problem of (5.1) as

$$
(L):\left\{\begin{array}{l}
\text { system }(6.2) \text { in } \omega_{2} \cup \omega_{3} \\
\text { boundary conditions (6.4) and } \delta \mu=0 \text { on } \Gamma_{i} \quad(i=2,3) \\
\delta p, \delta W \text { are continuous on } \Gamma_{D} \\
\delta p=0 \text { on } \Gamma_{L}
\end{array}\right.
$$

The whole linear problem $(L)$ can be separated into the following two subproblems:

$$
\begin{aligned}
& \left(L_{e}\right):\left\{\begin{array}{l}
D_{R} \delta W-e D_{I} \delta p+H_{1 \mathbf{U}} \delta \mathbf{U}=f_{1} \text { in } \omega, \\
D_{I} \delta W+e D_{R} \delta p+H_{2 \mathbf{U}} \delta \mathbf{U}=f_{2} \text { in } \omega, \\
\delta W+\tau_{W_{i}} \delta p=g_{W_{i}}, \text { on } \Gamma_{i}(i=2,3), \\
\delta p, \delta W \text { are continuous on } \Gamma_{D}, \\
\delta p=0 \text { on } \Gamma_{L},
\end{array}\right. \\
& \left(L_{h}\right):\left\{\begin{array}{l}
\frac{\partial}{\partial x} \delta U+d_{W} \frac{\partial}{\partial x} \delta W+d_{p} \frac{\partial}{\partial x} \delta p=f_{3} \text { in } \omega, \\
\frac{\partial}{\partial x} \delta p-\frac{\gamma p}{\rho} \frac{\partial}{\partial x} \delta \rho=f_{4} \text { in } \omega, \\
U \frac{\partial}{\partial x} \delta \mu-2 \delta \mu=f_{5} \text { in } \omega, \\
\delta U+\tau_{U_{i}} \delta p=g_{U_{i}} \text { on } \Gamma_{i}(i=2,3), \\
\delta \rho+\tau_{\rho_{i}} \delta p=g_{\rho_{i}} \text { on } \Gamma_{i}(i=2,3), \\
\delta \mu=0 \text { on } \Gamma_{i}(i=2,3) .
\end{array}\right.
\end{aligned}
$$

\section{Estimates FOR THE LINEARIZED PROBLEM}

7.1. $H^{k}$ estimates. In this section we are going to solve the linearized problem $(L)$ and give some estimates, which are useful for establishing the existence of the solution to the nonlinear problem $(N L)$. Since the problem $(L)$ is defined in $\omega$, which has piecewise smooth boundary with corners, and the coefficients of $L$ have discontinuity across the line $\Gamma_{D}$, then to obtain the estimates to its solution, we will use the results for elliptic equations in a domain with corners in [17, as well as the classical elliptic estimates in 18 . To this end we first make a coordinate 
transformation here to let the domain $\omega$ be symmetric with respect to $y=0$. The transformation is

$$
\Pi: \tilde{x}=x, \quad \tilde{y}= \begin{cases}-\frac{y}{\chi_{2}(x)} x & \text { if } y<0, \\ \frac{y}{\chi_{3}(x)} x & \text { if } \quad y>0 .\end{cases}
$$

Next we denote the image of $\omega_{2}, \omega_{3}$ under such transformation by $\omega_{-}, \omega_{+}$, the image of $\Gamma_{2}, \Gamma_{3}$ by $\Gamma_{-}, \Gamma_{+}$, and still denote the variables by $x, y$. For our convenience we first study the problems $\left(L_{e}\right)$ and $\left(L_{h}\right)$ without zero-order terms $H_{i \mathbf{U}} \delta \mathbf{U}$. Hence $\left(L_{e}\right)$ becomes

$$
\left(L_{e}^{1}\right):\left\{\begin{array}{l}
D_{R} \delta W-e D_{I} \delta p=f_{1} \text { in } \omega_{ \pm} \\
D_{I} \delta W+e D_{R} \delta p=f_{2} \text { in } \omega_{ \pm} \\
\delta W+\tau_{ \pm} \delta p=g_{W \pm} \text { on } \Gamma_{ \pm} \\
\delta p_{+}=\delta p_{-}, \delta W_{+}=\delta W_{-} \text {on } \Gamma_{D} \\
\delta p=0 \text { on } \Gamma_{L},
\end{array}\right.
$$

where $\tau_{ \pm}$and $g_{W \pm}$ are the image of $\tau_{W_{i}}$ and $g_{W_{i}}$ and where $(\delta p)_{ \pm}\left(\right.$resp. $\left.(\delta W)_{ \pm}\right)$ are the traces on $\Gamma_{D}$ of $\delta p$ (resp. $\left.\delta W\right)$ defined in $\Omega_{ \pm}$. The two differential equations in $\left(L_{e}^{1}\right)$ form an elliptic system with coefficients in $C^{1, \alpha}\left(\bar{\omega}_{ \pm}\right)$. Next we are going to derive a priori estimates in $H^{1}(\omega)$ for the problem $\left(L_{e}^{1}\right)$ and then solve it afterwards.

Lemma 7.1. Assume that $\left(\mathbf{U}_{0}, \mathbf{U}_{1}\right)$ belongs to an $\epsilon$-neighborhood of $\left(\mathbf{U}_{0}^{0}, \mathbf{U}_{1}^{0}\right)$ as shown in (2.19), $\left(\chi_{2}(x), \chi_{3}(x)\right) \in K_{\zeta},\left(U_{2}, U_{3}\right) \in \Sigma_{\delta}$ with sufficiently small $\epsilon, \zeta, \delta$. Assume that $(\delta p, \delta W) \in H^{1}(\omega)$ is the solution of (7.2), $f_{1,2} \in L^{2}(\omega), g_{W \pm} \in$ $H^{\frac{1}{2}}\left(\Gamma_{ \pm}\right)$. Then

$$
\|\delta p\|_{H^{1}(\omega)}^{2}+\|\delta W\|_{H^{1}(\omega)}^{2} \leq C\left(\sum_{i=1,2}\left\|f_{i}\right\|_{L^{2}(\omega)}^{2}+\sum_{+,-}\left\|g_{W \pm}\right\|_{H^{\frac{1}{2}\left(\Gamma_{ \pm}\right)}}^{2}\right)
$$

where $C$ is independent of $\epsilon, \zeta$ and $\delta$.

Proof. By subtracting a given function, we can only consider problem (7.2) with homogeneous boundary condition $g_{W \pm}=0$. Moreover, by using a standard regularization argument, $(\delta p, \delta W)$ can be assumed to be $C^{2}$ smooth in $\bar{\omega}$ in the process of the proof, because any $H^{1}(\omega)$ function can be approximated by $C^{2}(\bar{\omega})$ functions in $H^{1}(\omega)$, if the boundary of $\omega$ is piecewise smooth and the angles formed by the boundary $\partial \omega$ at all corners are bounded away from 0 and $\pi$. Multiplying the first equation of (7.2) by $D_{R} \delta W-D_{I} \delta p$ and multiplying the second equation of (7.2) by $D_{I} \delta W+D_{R} \delta p$ and then adding these terms, we obtain

$$
\begin{aligned}
& \left(D_{R} \delta W\right)^{2}+\left(D_{I} \delta W\right)^{2}+e\left(D_{I} \delta p\right)^{2}+e\left(D_{R} \delta p\right)^{2} \\
& \quad+(1+e)\left(D_{R} \delta p \cdot D_{I} \delta W-D_{R} \delta W \cdot D_{I} \delta p\right)=F
\end{aligned}
$$

where $F=f_{1}\left(D_{R} \delta W-D_{I} \delta p\right)+f_{2}\left(D_{I} \delta W+D_{R} \delta p\right)$. Multiplying $\frac{1}{\lambda_{I}(1+e)}$ and then integrating this equality on $\omega$ give us

$$
\begin{aligned}
& \iint_{\omega} \frac{1}{\lambda_{I}(1+e)}\left(\left(D_{R} \delta W\right)^{2}+\left(D_{I} \delta W\right)^{2}+e\left(D_{I} \delta p\right)^{2}+e\left(D_{R} \delta p\right)^{2}\right) d x d y \\
& \quad+\iint_{\omega} \frac{1}{\lambda_{I}}\left(D_{I} \delta W \cdot D_{R} \delta p-D_{I} \delta p \cdot D_{R} \delta W\right) d x d y=\iint_{\omega} \frac{F}{\lambda_{I}(1+e)} d x d y .
\end{aligned}
$$


Since $\lambda_{I}(1+e)>0$, we have

$$
\begin{aligned}
\|(\delta W, \delta p)\|_{H^{1}}^{2} \leq & \left|\iint_{\omega} \frac{1}{\lambda_{I}}\left(D_{I} \delta W \cdot D_{R} \delta p-D_{I} \delta p \cdot D_{R} \delta W\right) d x d y\right| \\
& +\iint_{\omega} \frac{F}{\lambda_{I}(1+e)} d x d y .
\end{aligned}
$$

Next we compute the first term in the right hand side of (7.4) by integrating by parts on $\omega_{ \pm}$separately. Noticing $D_{R}=\partial_{x}+\lambda_{R} \partial_{y}, D_{I}=\lambda_{I} \partial_{y}$, we have

$$
\begin{aligned}
& \iint_{\omega_{ \pm}} \frac{1}{\lambda_{I}}\left(D_{I} \delta W \cdot D_{R} \delta p-D_{I} \delta p \cdot D_{R} \delta W\right) d x d y \\
& =\iint_{\omega_{ \pm}} \frac{1}{\lambda_{I}}\left(D_{R}\left(\delta p \cdot D_{I} \delta W\right)-D_{I}\left(\delta p \cdot D_{R} \delta W\right)\right) d x d y+\iint_{\omega_{ \pm}} R_{1} d x d y \\
& =\int_{\partial \omega_{ \pm}}\left(\frac{1}{\lambda_{I}} \delta p \cdot D_{I} \delta W \cos (n, x)+\frac{\lambda_{R}}{\lambda_{I}} \delta p \cdot D_{I} \delta W \cos (n, y)-\right. \\
& \left.\quad \delta p \cdot D_{R} \delta W \cos (n, y)\right) d s+\iint_{\omega_{ \pm}} R_{1} d x d y,
\end{aligned}
$$

where $R_{1}$ stands for the terms satisfying

$$
\left\|R_{1}\right\|_{L^{1}(\omega)} \leq C(\delta+\zeta)\|(\delta p, \delta W)\|_{L^{2}(\omega)} \cdot\|(\delta p, \delta W)\|_{H^{1}(\omega)},
$$

which can be different in different equalities .

The integral on $\partial \omega_{ \pm}$in (7.5) equals

$$
\begin{aligned}
\int_{\partial \omega_{ \pm}} & \delta p \cdot \partial_{y} \delta W \cos (n, x)-\delta p \cdot \partial_{x} \delta W \cos (n, y) \\
= & \int_{\partial \omega_{ \pm}} \delta p \cdot \partial_{s} \delta W d s .
\end{aligned}
$$

The sum of the integrals on $\partial \omega_{+}$and $\partial \omega_{-}$is a sum of the corresponding integrals on $\Gamma_{L}, \Gamma_{ \pm}$and $\Gamma_{D}$. The integral on $\Gamma_{L}$ vanishes due to $\delta p=0$ on it. The corresponding integrals on $\Gamma_{D}$ are cancelled because of the continuity of $\delta p$ and $\delta W$. Therefore, we only need to compute the integral on $\Gamma_{ \pm}$.

Letting the tangential differential operator $\partial_{s}$ act on the boundary condition on $\Gamma_{ \pm}$in (7.2) with $g_{w \pm}=0$, we have

$$
\partial_{s} \delta W+\tau_{ \pm} \partial_{s} \delta p=-\left(\partial_{s} \tau_{ \pm}\right) \delta p
$$

Substituting it into the integral along $\partial \omega_{ \pm}$, we obtain

$$
\begin{aligned}
& \int_{\Gamma_{ \pm}} \delta p \cdot \partial_{s} \delta W d s=\int_{\Gamma_{ \pm}}\left(-\tau_{ \pm} \delta p \partial_{s} \delta p-\left(\partial_{s} \tau_{ \pm}\right) \delta p^{2}\right) d s \\
& =\int_{\Gamma_{ \pm}} \partial_{s}\left(-\frac{1}{2} \tau_{ \pm} \delta p^{2}\right) d s-\int_{\Gamma_{ \pm}} \frac{1}{2}\left(\partial_{s} \tau_{ \pm}\right) \delta p^{2} d s .
\end{aligned}
$$

The sum of the first term on $\Gamma_{+}$and $\Gamma_{-}$vanishes, because $\delta p$ is continuous on the vertex of $\omega$, while the second term is dominated by $O(\delta)\|\delta p\|_{H^{1}(\omega)}^{2}$. Combining the equalities from (7.4) to (7.7) and noticing the estimate for $R_{1}$, we are led to (7.3). 
Remark 7.1. The conclusion in Lemma 7.2 is still valid, if the differential system in (7.2) is replaced by

$$
\left\{\begin{array}{l}
D_{R} \delta W-e D_{I} \delta p+\gamma_{11} \delta W+\gamma_{12} \delta p=f_{1} \\
D_{I} \delta W+e D_{R} \delta p+\gamma_{21} \delta W+\gamma_{22} \delta p=f_{2}
\end{array}\right.
$$

We notice that the lower order terms do not influence the ellipticity of the systems. Then the statement in the proof of Lemma 7.1 can also be applied here. The only difference is that the term $R_{1}$ in (7.5) could be changed, but still satisfy the estimate (7.6).

Lemma 7.2. Under the assumptions of Lemma 7.1, if $(\delta p, \delta W) \in H^{2}\left(\omega_{ \pm}\right) \cap H^{1}(\omega)$ is the solution of (7.2), $\left.f_{1,2}\right|_{\omega_{ \pm}} \in H^{1}\left(\omega_{ \pm}\right), g_{W \pm} \in H^{\frac{3}{2}}\left(\Gamma_{ \pm}\right)$, then

$$
\|\delta p\|_{H^{2}\left(\omega_{ \pm}\right)}^{2}+\|\delta W\|_{H^{2}\left(\omega_{ \pm}\right)}^{2} \leq C \sum_{+,-}\left(\left\|f_{1,2}\right\|_{H^{1}\left(\omega_{ \pm}\right)}^{2}+\left\|g_{W \pm}\right\|_{H^{\frac{3}{2}\left(\Gamma_{ \pm}\right)}}^{2}\right),
$$

where $C$ is independent of $\epsilon, \zeta$ and $\delta$.

Proof. Similarly to Lemma 7.1 we can only consider (7.2) with homogeneous boundary condition $g_{W \pm}=0$.

Let us first indicate that for any function $u \in H^{1}(\omega)$ satisfying $\left.u\right|_{\omega_{ \pm}} \in H^{2}\left(\omega_{ \pm}\right)$, there is a sequence $u_{\epsilon} \in C^{0}(\bar{\omega})$, satisfying $\left.u_{\epsilon}\right|_{\bar{\omega}_{ \pm}} \in C^{2}\left(\bar{\omega}_{ \pm}\right)$, such that

$$
u_{\epsilon} \longrightarrow u\left(H^{1}(\omega)\right),\left.\left.\quad u_{\epsilon}\right|_{\omega_{ \pm}} \longrightarrow u\right|_{\omega_{ \pm}}\left(H^{2}\left(\omega_{ \pm}\right)\right) \text {as } \epsilon \longrightarrow 0 .
$$

Indeed, according to the Sobolev imbedding theorem, $u(x, 0) \in H^{\frac{3}{2}}\left(\Gamma_{D}\right)$. Let $v(x, y)$ be an $H^{2}(\omega)$ function taking trace on $\Gamma_{D}: \gamma_{0} v=u(x, 0), \gamma_{1} v=\gamma_{0}\left(\frac{\partial v}{\partial y}\right)=0$. Then by subtracting $v(x, y)$, we are reduced to discussing the case $u(x, 0)=0$. Therefore, let

$$
u_{+}(x, y)=\left\{\begin{array}{l}
u(x, y), y>0, \\
-u(x,-y), \quad y<0,
\end{array} \quad u_{-}(x, y)=\left\{\begin{array}{l}
-u(x,-y), y>0, \\
u(x, y), y<0 .
\end{array}\right.\right.
$$

Both $u_{ \pm}(x, y)$ belong to $H^{2}(\omega)$. Now define

$$
u_{ \pm \epsilon}(x, y)=\int j_{\epsilon}\left(x^{\prime}, y^{\prime}\right) u_{ \pm}\left(x-x^{\prime}, y-y^{\prime}\right) d x^{\prime} d y^{\prime}
$$

where $j_{\epsilon}$ is a $C^{\infty}$ kernel of mollifier, taking weighted average for $u_{ \pm}$in an $\epsilon$-size domain $O_{\epsilon} \subset \omega$, which is symmetric with respect to $y^{\prime}$. Then let $u_{\epsilon}(x, y)$ equal $u_{ \pm \epsilon}(x, y)$ in $\omega_{ \pm}$. We have

$$
\begin{aligned}
& \left.u_{\epsilon}\right|_{\bar{\omega}_{ \pm}} \in C^{\infty}\left(\bar{\omega}_{ \pm}\right), u_{\epsilon}(x, 0)=0, \\
& \left\|u_{\epsilon}-u\right\|_{H^{2}\left(\omega_{ \pm}\right)} \longrightarrow 0 .
\end{aligned}
$$

Moreover, due to the continuity on $y=0$, we also have

$$
u_{\epsilon} \in C^{0}(\bar{\omega}) \cap H^{1}(\omega), \quad\left\|u_{\epsilon}-u\right\|_{H^{1}(\omega)} \longrightarrow 0 .
$$

Based on the above discussion we can only consider the case $(\delta p, \delta W) \in C^{2}\left(\bar{\omega}_{ \pm}\right) \cap$ $C^{0}(\bar{\omega})$ in the proof of this lemma. Differentiating the first two equations in $\left(L_{e}^{1}\right)$ with respect to $x$ and denoting $\partial_{x} \delta p, \partial_{x} \delta W$ by $\widehat{\delta p}, \widehat{\delta W}$, we have

$$
\left\{\begin{array}{l}
D_{R} \widehat{\delta W}-e D_{I} \widehat{\delta p}=F_{1} \\
D_{I} \widehat{\delta W}+e D_{R} \widehat{\delta p}=F_{2}
\end{array}\right.
$$


where $F_{1}=\partial_{x} f_{1}-\partial_{x} \lambda_{R} \cdot \partial_{y} \delta W+\partial_{x} e \cdot D_{I} \delta p+e \partial_{x} \lambda_{I} \cdot \partial_{y} \delta p, F_{2}=\partial_{x} f_{2}-\partial_{x} \lambda_{I}$. $\partial_{y} \delta W-\partial_{x} e \cdot D_{R} \delta p-e \partial_{x} \lambda_{R} \cdot \partial_{y} \delta p$. Obviously, by virtue of the assumptions of the lemma, we have $F_{1,2} \in L^{2}(\omega)$.

The boundary conditions for $\widehat{\delta p}$ and $\widehat{\delta W}$ can be derived as follows. On $\Gamma_{L}, \delta p=0$ implies $\partial_{y} \delta p=0$. Then by using two equations in $\left(L_{e}\right)$ and $\left.\partial_{y} \delta p\right|_{\Gamma_{L}}=0$, we obtain

$$
\lambda_{I} \widehat{\delta W}-e \lambda_{R} \widehat{\delta p}=\lambda_{I} f_{1}-\lambda_{R} f_{2} \text { on } \Gamma_{L}
$$

On $\Gamma_{ \pm}$, regarding the equations in $\left(L_{e}^{1}\right)$ as linear combinations of $\partial_{x} \delta p, \partial_{y} \delta p$, $\partial_{x} \delta W$ and $\partial_{y} \delta W$, the coefficients matrix is

$$
\left(\begin{array}{cccc}
0 & -e \lambda_{I} & 1 & \lambda_{R} \\
e & e \lambda_{R} & 0 & \lambda_{I}
\end{array}\right)
$$

Obviously, its sub-matrix formed by the second and fourth columns is nonsingular. Then we can express $\partial_{y} \delta p$ and $\partial_{y} \delta W$ by a linear combination of $\widehat{\delta p}$ and $\widehat{\delta W}$. On the other hand, by differentiating the boundary condition on $\Gamma_{ \pm}$along the boundary, which has direction $(1, \pm 1)$, we obtain

$$
\partial_{x} \delta W \pm \partial_{y} \delta W+\partial_{x}\left(\tau_{ \pm} \delta p\right) \pm \partial_{y}\left(\tau_{ \pm} \delta p\right)=\partial_{x} g_{W \pm} \pm \partial_{y} g_{W \pm} .
$$

Substituting the expression of $\partial_{y} \delta p$ and $\partial_{y} \delta W$ into this equality, we obtain

$$
\widehat{\delta W}+\hat{\tau}_{ \pm} \widehat{\delta p}=\hat{k}_{ \pm} \delta p+\hat{g}_{W \pm}
$$

Finally, in view of the fact that the differential operator $\partial_{x}$ is along the tangential direction of $\Gamma_{D}$, we also have

$$
(\widehat{\delta p})_{+}=(\widehat{\delta p})_{-}, \quad(\widehat{\delta W})_{+}=(\widehat{\delta W})_{-} \text {on } \Gamma_{D}
$$

in the sense of trace. Equalities (7.11)-(7.14) form a boundary value problem for $(\widehat{\delta p}, \widehat{\delta W})$. Similarly to the discussion on $\left(L_{e}^{1}\right)$ in the previous lemma, we can also establish the estimate of the $H^{1}$ norm for $\widehat{\delta p}$ and $\widehat{\delta W}$. That is

$$
\begin{gathered}
\left\|\left(\partial_{x} \delta p, \partial_{x} \delta W\right)\right\|_{H^{1}\left(\omega_{ \pm}\right)}^{2} \leq C\left(\sum_{i=1,2}\left\|F_{i}\right\|_{L^{2}(\omega)}^{2}+\sum_{+,-}\left\|\hat{k}_{ \pm} \delta p+\hat{g}_{W \pm}\right\|_{H^{\frac{1}{2}}\left(\Gamma_{ \pm}\right)}^{2}\right) \\
\leq C \sum_{+,-} \sum_{i=1,2}\left\|F_{i}\right\|_{H^{1}\left(\omega_{ \pm}\right)}^{2} .
\end{gathered}
$$

Once again using the equations in $\left(L_{e}^{1}\right)$, we obtain the same estimate for $\left\|\left(\partial_{y} \delta p, \partial_{y} \delta W\right)\right\|_{H^{1}\left(\omega_{+}\right)}^{2}$ with different $C$. Going back to the case with nonhomogeneous boundary condition, we obtain (7.9).

According to the classical theory of elliptic equations [1], we can derive the unique existence of the solution in $H^{1}(\omega)$ for the problem $\left(L_{e}^{1}\right)$ from (7.3) and $H^{2}\left(\omega_{ \pm}\right)$regularity of the solution from (7.9). Hence we have

Theorem 7.1. Assume that $\left(\mathbf{U}_{0}, \mathbf{U}_{1}\right) \in N_{\epsilon},\left(S_{2}, S_{3}\right) \in K_{\zeta},\left(U_{2}, U_{3}\right) \in \Sigma_{\delta}$ with $\epsilon, \eta, \delta$ being sufficiently small, $f_{1}, f_{2} \in L^{2}(\omega), g_{W \pm} \in H^{\frac{1}{2}}\left(\Gamma_{ \pm}\right)$. Then the problem $\left(L_{e}^{1}\right)$ admits an $H^{1}(\omega)$ solution $(\delta p, \delta W)$ in $\omega$. Moreover, the solution belongs to $H^{2}\left(\omega_{ \pm}\right)$, and the estimates (7.3), (7.10) hold.

Remark 7.2. As in the statement in Remark 7.1, the conclusion in Theorem 7.1 is still valid, if the differential system in (7.2) is replaced by (7.10). 
7.2. $C^{1, \alpha}$ estimates. Now we establish piecewise $C^{1, \alpha}$ estimates for the solution to $\left(L_{e}^{1}\right)$. Since the domain $\omega_{ \pm}$does not have smooth boundary, we should use the results in [5, 6, 16, 17, on elliptic boundary value problem in curvilinear polygons. To this end we first reduce $\left(L_{e}^{1}\right)$ to a boundary value problem of second order elliptic equations.

Denote by $[\cdot, \cdot]$ the Poisson bracket. We have

$$
\left[D_{R}, D_{I}\right]=\mu D_{I},
$$

where $\mu=\frac{1}{\lambda_{I}}\left(\frac{\partial \lambda_{I}}{\partial x}+\lambda_{R} \frac{\partial \lambda_{I}}{\partial y}-\lambda_{I} \frac{\partial \lambda_{R}}{\partial y}\right)$ satisfies $\|\mu\|_{C^{1, \alpha}} \leq C \delta_{0}$.

Applying $D_{I}$ and $D_{R}$ in the first two equations in (7.2), respectively, and then subtracting the first one from the second one, we obtain

$$
D_{I}\left(e D_{I} \delta p\right)+D_{R}\left(e D_{R} \delta p\right)+\left[D_{R}, D_{I}\right] \delta W=D_{R} f_{2}-D_{I} f_{1} .
$$

Substituting (7.16) into (7.17), we obtain a second order elliptic system with divergence form

$$
D_{I}\left(e D_{I} \delta p\right)+D_{R}\left(e D_{R} \delta p\right)-\mu e D_{R} \delta p=f^{*},
$$

where $f^{*}=D_{R} f_{2}-D_{I} f_{1}-\mu f_{2}$.

Differentiating the boundary condition in (7.2) along $\Gamma_{ \pm}$gives us

$$
\partial_{s} \delta W+\left(\partial_{s} \tau_{ \pm}\right) \delta p+\tau_{ \pm} \partial_{s} \delta p=\partial_{s} g_{W \pm}
$$

where $\partial_{s}$ is the differential operator along $\Gamma_{ \pm}$. By using the equations in (7.2), we have

$$
\begin{aligned}
& \partial_{s} \delta W=\left(\partial_{x}+\lambda_{R} \partial_{y}\right) \delta W+\frac{\left( \pm 1-\lambda_{R}\right)}{\lambda_{I}} \lambda_{I} \partial_{y} \delta W \\
& =\left(e D_{I} \delta p+f_{1}\right)+\frac{\left( \pm 1-\lambda_{R}\right)}{\lambda_{I}}\left(-e D_{R} \delta p+f_{2}\right)
\end{aligned}
$$

on $\Gamma_{ \pm}$. Meanwhile,

$$
\partial_{s} \delta p=D_{R} \delta p+\frac{\left( \pm 1-\lambda_{R}\right)}{\lambda_{I}} D_{I} \delta p
$$

Hence, we have

$$
\left(\chi_{1 \pm} \frac{\partial}{\partial x}+\chi_{2 \pm} \frac{\partial}{\partial y}+\chi_{3 \pm}\right) \delta p=h_{ \pm}, \quad \text { on } \quad \Gamma_{ \pm}
$$

where

$$
\begin{gathered}
\chi_{1 \pm}=-\frac{1}{\lambda_{I}}\left( \pm 1-\lambda_{R}\right) e+\tau_{ \pm}, \\
\chi_{2 \pm}=\left(\lambda_{I}-\frac{\lambda_{R}}{\lambda_{I}}\left( \pm 1-\lambda_{R}\right)\right) e \pm \tau_{ \pm}, \\
\chi_{3 \pm}=\partial_{s} \tau_{ \pm}, \\
h_{ \pm}=\partial_{s} g-f_{1}-\frac{1}{\lambda_{I}}\left( \pm 1-\lambda_{R}\right) f_{2} .
\end{gathered}
$$

Consider the condition on $\Gamma_{D}$. By eliminating $\partial_{y} \delta W$ from the equations in $\left(L_{e}^{1}\right)$, we have

$$
\partial_{x} \delta W=\frac{e}{\lambda_{I}}\left(\lambda_{R} \partial_{x} \delta p+\left(\lambda_{R}^{2}+\lambda_{I}^{2}\right) \partial_{y} \delta p\right)+\lambda_{I} f_{1}-\lambda_{R} f_{2},
$$


which is continuous on $\Gamma_{D}$. Therefore, $\delta p$ is a solution to the problem of a second order elliptic equation in $\omega$ as follows.

$$
\left(L_{e}^{2}\right):\left\{\begin{array}{l}
D_{I}\left(e D_{I} \delta p\right)+D_{R}\left(e D_{R} \delta p\right)-\mu e D_{R} \delta p=f^{*}, \quad \text { in } \omega_{ \pm}, \\
\left(\chi_{1 \pm} \frac{\partial}{\partial \xi}+\chi_{2 \pm} \frac{\partial}{\partial \eta}+\chi_{3 \pm}\right) \delta p=h_{ \pm}, \quad \text { on } \Gamma_{ \pm}, \\
\delta p \text { and } \frac{e}{\lambda_{I}}\left(\lambda_{R} \partial_{x} \delta p+\left(\lambda_{R}^{2}+\lambda_{I}^{2}\right) \partial_{y} \delta p\right)+\lambda_{I} f_{1}-\lambda_{R} f_{2} \\
\delta p=0, \quad \text { on } \Gamma_{L} .
\end{array}\right.
$$

For the problem $\left(L_{e}^{2}\right)$ we introduce a reflection transformation, which folds the whole domain $\omega=\omega_{+} \cup \omega_{-}$to the upper half part $\omega_{+}$. Let $\widetilde{\delta p}=\left(\widetilde{\delta p_{1}}, \widetilde{\delta p_{2}}\right)$ with $\widetilde{\delta p_{1}}(x, y)=\delta p(x, y), \widetilde{\delta p_{2}}(x, y)=\delta p(x,-y)$. Then (7.20) is reduced to a new problem for $\widetilde{\delta p}$ in $\omega_{+}$as

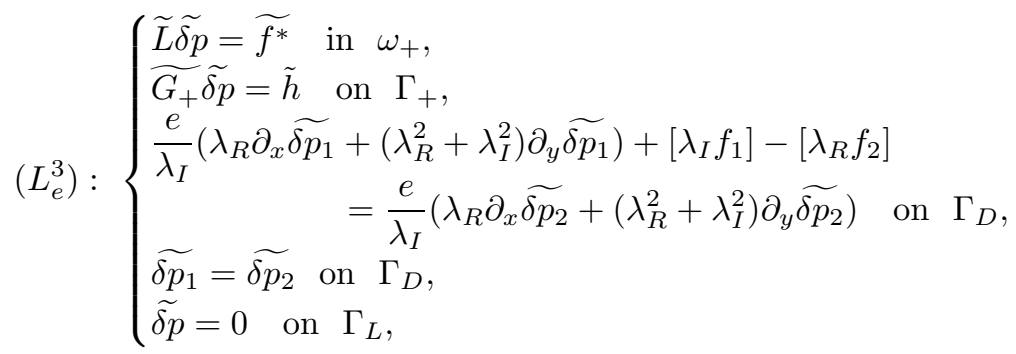

where

$$
\widetilde{f^{*}}(x, y)=\left(\begin{array}{c}
f^{*}(x, y) \\
f^{*}(x,-y)
\end{array}\right), \quad \tilde{h}(x, y)=\left(\begin{array}{c}
h_{+}(x, y) \\
h_{-}(x,-y)
\end{array}\right)
$$

and the explicit form of operators $\tilde{L}$ and $\tilde{G}_{+}$can be simply obtained from (7.20). It is easy to check (see [21]) that the boundary conditions for the problem $\left(L_{e}^{3}\right)$ on $\Gamma_{D}, \Gamma_{+}$and $\Gamma_{L}$ are "complementing boundary conditions" in the sense of [1, 2]. Therefore, by using the results in [6, 17, 18, the solution of the problem $\left(L_{e}^{3}\right)$ can be decomposed as a sum of a regular part and a singular part. More precisely, if $\widetilde{f^{*}} \in C^{\sigma}\left(\bar{\omega}_{+}\right), \widetilde{g} \in C^{1, \sigma}\left(\Gamma_{+}\right)$and the coefficients in (7.21) are $C^{2, \sigma}\left(\bar{\omega}_{+}\right)$, then the solution of (7.21) can be written as

$$
\begin{aligned}
& \widetilde{\delta p}=\widetilde{\delta p}_{r}+\widetilde{\delta p}_{s} \\
& \widetilde{\delta p}_{r} \in C^{2, \sigma}, \quad \widetilde{\delta p}_{s}=\sum_{j, m} c_{j, m} S_{j, m},
\end{aligned}
$$

with

$$
S_{j, m}=\left\{\begin{array}{l}
r_{j}^{\lambda_{j, m}} \phi_{j, m}\left(\theta_{j}\right), \text { if } \lambda_{j, m} \text { is not an integer, } \\
r_{j}^{\lambda_{j, m}}\left(\log r_{j} \phi_{j, m}\left(\theta_{j}\right)+\psi_{j, m}\left(\theta_{j}\right)\right), \text { if } \lambda_{j, m} \text { is an integer, }
\end{array}\right.
$$

where $\left(r_{j}, \theta_{j}\right)$ are the local polar coordinates in the neighborhood of the $j$-th vertex of the polygon, $\lambda_{j, m}>0$ is the eigenvalue of an auxiliary boundary value problem of ordinary differential equations introduced from (7.21), $\phi_{j, m}, \psi_{j, m}$ are $C^{2, \sigma}$ functions of $\theta_{j}$. Moreover, if $\widetilde{\delta p}$ is a $C^{1, \sigma}$ solution of (17.21), then

$$
\|\widetilde{\delta p}\|_{C^{1, \sigma}\left(\bar{\omega}_{+}\right)} \leq C\left(\left\|f_{1,2}\right\|_{C^{\sigma}\left(\bar{\omega}_{+}\right)}+\|\widetilde{h}\|_{C^{\sigma}\left(\Gamma_{+}\right)}\right),
$$


where the maximum principle and the expression $\widetilde{f^{*}}$ have been applied.

Since Theorem 7.1 indicates $\widetilde{\delta p} \in H^{2}\left(\omega_{+}\right)$, then we must have

$$
\min \lambda_{j, m}>1 \text {. }
$$

By taking $\alpha^{\prime}=\min \left(\alpha,\left(\min \lambda_{j, m}-1\right) / 2\right)$, where $\alpha$ is given in Theorem 2.1, we have $(\widetilde{\delta p})_{s} \in C^{1, \alpha^{\prime}}$. Therefore, $\widetilde{\delta p}=(\widetilde{\delta p})_{r}+(\widetilde{\delta p})_{s} \in C^{1, \alpha^{\prime}}\left(\bar{\omega}_{+}\right)$, and the $C^{1, \alpha^{\prime}}$ estimate for $\widetilde{\delta p}$ as in (7.24) with $\alpha^{\prime}$ replacing $\sigma$ holds. Substituting the expression of $\widetilde{f^{*}}$ and $\tilde{g}$, we obtain

$$
\|\widetilde{\delta p}\|_{C^{1, \alpha^{\prime}}\left(\bar{\omega}_{+}\right)} \leq C \sum_{+,-}\left(\left\|f_{1,2}\right\|_{C^{\alpha^{\prime}\left(\bar{\omega}_{ \pm}\right)}}+\left\|g_{W \pm}\right\|_{C^{1, \alpha^{\prime}}\left(\Gamma_{ \pm}\right)}\right) .
$$

Going back to the solution $\delta p$ of the problem $\left(L_{e}^{2}\right)$ and the solution $(\delta p, \delta W)$ of the problem $\left(L_{e}^{1}\right)$, we know that the estimate (7.26) is also valid for the solution $(\delta p, \delta W)$ of $\left(L_{e}^{1}\right)$. Since $\lambda_{j, m}$ is independent of $\alpha$, we can simply take the index $\alpha_{0}$ defined in (2.14) larger than $\left(\min \lambda_{j, m}-1\right) / 2$ and let $\alpha^{\prime}=\alpha=\left(\min \lambda_{j, m}-1\right) / 2$. Then we obtain

Theorem 7.2. Under the assumptions of Theorem 7.1 and with $f_{1}, f_{2} \in C^{\alpha}\left(\bar{\omega}_{ \pm}\right)$, $g_{W \pm} \in C^{1, \alpha}\left(\Gamma_{ \pm}\right)$, the problem $\left(L_{e}^{1}\right)$ admits a solution $(\delta p, \delta W)$, which is continuous in $\bar{\omega}$, is $C^{1, \alpha}$ in $\bar{\omega}_{ \pm}$, and satisfies

$$
\|(\delta p, \delta W)\|_{C^{1, \alpha}\left(\bar{\omega}_{ \pm}\right)} \leq C \sum_{+,-}\left(\left\|f_{1,2}\right\|_{C^{\alpha}\left(\bar{\omega}_{ \pm}\right)}+\left\|g_{W \pm}\right\|_{C^{1, \alpha}\left(\Gamma_{ \pm}\right)}\right),
$$

where $C$ is independent of $\epsilon, \zeta$ and $\delta$.

Remark 7.3. Like the statement in Remark 7.1, the conclusion in Theorem 7.2 is still valid, if the differential system in (7.2) is replaced by (7.8). Indeed, according to the above reduction such a replacing only changes the lower order terms of the second order elliptic equation (7.18). Obviously, such a change does not influence the latter argument to derive the estimate (7.27).

7.3. Existence and estimates of the solution to problem $(L)$. Having obtained the solution $(\delta p, \delta W)$ of $\left(L_{e}^{1}\right)$ and its estimates, we can further integrate $\left(L_{h}\right)$. In (6.9) the coefficients of $\frac{\partial \delta U}{\partial x}, \frac{\partial \delta \rho}{\partial x}, \frac{\partial \delta \mu}{\partial x}$ are away from zero, so that $\left(L_{h}\right)$ can be simply integrated, and then the estimates for $\delta U, \delta \rho, \delta \mu$ can be established.

$$
\begin{gathered}
\|(\delta U, \delta \rho, \delta \mu)\|_{C^{1, \alpha}\left(\bar{\omega}_{ \pm}\right)} \leq C \sum_{+,-}\left(\sum_{j=1,2}\left\|f_{j}\right\|_{C^{\alpha}\left(\bar{\omega}_{ \pm}\right)}+\sum_{j=3,4,5}\left\|f_{j}\right\|_{C^{1, \alpha}\left(\bar{\omega}_{ \pm}\right)}\right. \\
\left.+\left\|\left(g_{W_{ \pm}}, g_{U_{ \pm}}, g_{\rho_{ \pm}}\right)\right\|_{C^{1, \alpha}\left(\Gamma_{ \pm}\right)}\right),
\end{gathered}
$$

By using the inverse transformation of (7.1), we obtain

$$
\begin{aligned}
\|\delta \mathbf{U}\|_{C^{1, \alpha}\left(\bar{\omega}_{2,3}\right)} \leq C \sum_{i=2,3} & \left(\sum_{j=1,2}\left\|f_{j}\right\|_{C^{\alpha}\left(\bar{\omega}_{i}\right)}\right. \\
& \left.+\sum_{j=3,4,5}\left\|f_{j}\right\|_{C^{1, \alpha}\left(\bar{\omega}_{i}\right)}+\left\|\left(g_{W_{i}}, g_{U_{i}}, g_{\rho_{i}}\right)\right\|_{C^{1, \alpha}\left(\Gamma_{i}\right)}\right) .
\end{aligned}
$$

Now let us deal with the problems $\left(L_{e}\right)$ and $\left(L_{h}\right)$ with general zero-order terms $H_{i \mathbf{U}} \delta \mathbf{U}$. Write the equations in (6.8) as

$$
\left\{\begin{array}{l}
D_{R} \delta W-e D_{I} \delta p+H_{1 W} \delta W+H_{1 p} \delta p=f_{1}-H_{1 U} \delta U-H_{1 \rho} \delta \rho, \\
D_{I} \delta W+e D_{R} \delta p+H_{2 W} \delta W+H_{2 p} \delta p=f_{2}-H_{2 U} \delta U+H_{2 \rho} \delta \rho .
\end{array}\right.
$$


According to Theorem 7.2 and the Remark 7.3 we have the estimate

$$
\begin{aligned}
\|\delta \mathbf{U}\|_{C^{1, \alpha}\left(\bar{\omega}_{2,3}\right)} & \leq C \sum_{i=2,3}\left(\sum_{j=1,2}\left\|f_{j}-H_{j U} \delta U-H_{j \rho} \delta \rho\right\|_{C^{\alpha}\left(\bar{\omega}_{i}\right)}\right. \\
& \left.+\sum_{j=3,4,5}\left\|f_{j}\right\|_{C^{1, \alpha}\left(\bar{\omega}_{i}\right)}+\left\|\left(g_{W_{i}}, g_{U_{i}}, g_{\rho_{i}}\right)\right\|_{C^{1, \alpha}\left(\Gamma_{i}\right)}\right) .
\end{aligned}
$$

Since

$$
\left\|f_{j}-H_{j U} \delta U-H_{j \rho} \delta \rho\right\|_{C^{\alpha}\left(\bar{\omega}_{i}\right)} \leq\left\|f_{j}\right\|_{C^{\alpha}\left(\bar{\omega}_{i}\right)}+C \delta\|\delta U, \delta \rho\|_{C^{\alpha}\left(\bar{\omega}_{i}\right)} \quad(j=1,2),
$$

by taking $\delta$ sufficiently small, the a priori estimates (7.29) are also valid for the solutions to $\left(L_{e}\right),\left(L_{h}\right)$ with $H_{j} \neq 0$.

The existence of the problem $(L)$ can be easily derived from the a priori estimate and the existence of $\left(L_{e}\right),\left(L_{h}\right)$ via iteration. Therefore, we are led to the following proposition.

Theorem 7.3. Under the assumptions of Theorem 7.1, if $f_{1,2} \in C^{\alpha}\left(\bar{\omega}_{i}\right), f_{3,4,5} \in$ $C^{1, \alpha}\left(\bar{\omega}_{i}\right)$ and $g_{W_{i}}, g_{U_{i}}, g_{\rho_{i}} \in C^{1, \alpha}\left(\Gamma_{i}\right)$ with $i=2,3$, then the problem $(L)$ admits a solution $\delta \mathbf{U}$, which is in $H^{1}(\omega)$ and in $C^{1, \alpha}\left(\bar{\omega}_{2,3}\right)$. Moreover, the solution satisfies the estimate

$$
\begin{aligned}
\|\delta \mathbf{U}\|_{C^{1, \alpha}\left(\bar{\omega}_{2,3}\right)} \leq C \sum_{i=2,3}( & \sum_{j=1,2}\left\|f_{j}\right\|_{C^{\alpha}\left(\bar{\omega}_{i}\right)} \\
& \left.+\sum_{j=3,4,5}\left\|f_{j}\right\|_{C^{1, \alpha}\left(\bar{\omega}_{i}\right)}+\left\|\left(g_{W_{i}}, g_{U_{i}}, g_{\rho_{i}}\right)\right\|_{C^{1, \alpha}\left(\Gamma_{i}\right)}\right),
\end{aligned}
$$

where $C$ is independent of $\varepsilon, \zeta$ and $\delta$.

\section{Solution to Problem $(N L)$}

In this section we use the results on the linearized problem obtained above to solve problem $(N L)$ by applying iterative methods. Let $\mathbf{U}_{\ell}$ denote the value of $\mathbf{U}$ in the left hand side of $\Gamma_{2,3}$, and define

$$
\mathbf{U}^{(\mathbf{0})}=\mathbf{U}_{r}^{0}= \begin{cases}\mathbf{U}_{2}^{0} & \text { in } \omega_{2}, \\ \mathbf{U}_{3}^{0} & \text { in } \omega_{3} .\end{cases}
$$

We can construct $\left\{\mathbf{U}^{(n)}\right\}$ by induction. Indeed, if $\mathbf{U}^{(n)} \in \Sigma_{\delta}$ is obtained, then $\delta \mathbf{U}^{(n+1)}$ is defined by the following problem:

$$
L^{(n)}:\left\{\begin{array}{l}
D_{R}^{(n)} \delta W^{(n+1)}-e^{(n)} D_{I}^{n} \delta p^{(n+1)}+H_{1 \mathbf{U}}^{(n)} \delta \mathbf{U}^{(n+1)}=-H_{1}^{(n)}+H_{1 \mathbf{U}}^{(n)} \delta \mathbf{U}^{(n)}, \\
D_{I}^{(n)} \delta W^{(n+1)}+e^{(n)} D_{R}^{n} \delta p^{(n+1)}+H_{2 \mathbf{U}}^{(n)} \delta \mathbf{U}^{(n+1)}=-H_{2}^{(n)}+H_{2 \mathbf{U}}^{(n)} \delta \mathbf{U}^{(n)}, \\
\frac{\partial}{\partial x} \delta U^{(n+1)}+d_{W}^{(n)} \frac{\partial}{\partial x} \delta W^{(n+1)}+d_{p}^{(n)} \frac{\partial}{\partial x} \delta p^{(n+1)}=0, \\
\frac{\partial}{\partial x} \delta p^{(n+1)}-\left(\frac{\gamma p}{\rho}\right)^{(n)} \frac{\partial}{\partial x} \delta \rho^{(n+1)}=0, \\
U^{(n)} \frac{\partial}{\partial x} \delta \mu^{(n+1)}-2 \mu^{(n+1)}=0, \\
\left(G_{\mathbf{U}}^{\sharp}\right)^{(n)} \delta \mathbf{U}^{(n+1)}=-G^{\sharp}\left(\mathbf{U}_{\ell}, \mathbf{U}^{(n)}\right)+\left(G_{\mathbf{U}}^{\sharp}\right)^{(n)} \delta \mathbf{U}^{(n)} \quad \text { on } \Gamma_{2,3} \quad(\sharp=a, b, c), \\
\delta \mu^{(n+1)}=0 \text { on } \Gamma_{2,3}, \\
\delta p^{(n+1)}, \delta W^{(n+1)} \text { are continuous on } \Gamma_{D}, \\
\delta p^{(n+1)}=0 \quad \text { on } \Gamma_{L} .
\end{array}\right.
$$


Then we define $\mathbf{U}^{(n+1)}=\mathbf{U}^{(0)}+\delta \mathbf{U}^{(n+1)}$.

Theorem 8.1. Under the assumptions of Theorem 7.3, the solution $\left\{\delta \mathbf{U}^{(n+1)}\right\}$ to the problem (8.1) is well defined and is uniformly bounded in $C^{1, \alpha}\left(\bar{\omega}_{i}\right)$.

Proof. Since $\mathbf{U}_{\ell} \in N_{\varepsilon}$ and $G^{\sharp}\left(\mathbf{U}_{\ell}^{0}, \mathbf{U}_{r}^{0}\right)=0$, then for $i=2,3$

$$
\left\|G^{\sharp}\left(\mathbf{U}_{\ell}, \mathbf{U}_{r}^{0}\right)\right\|_{C^{1, \alpha}\left(\Gamma_{i}\right)} \leq C \varepsilon .
$$

Write

$$
\begin{aligned}
\left(g^{\sharp}\right)^{(n)} & \triangleq-G^{\sharp}\left(\mathbf{U}_{\ell}, \mathbf{U}^{(n)}\right)+\left(G_{\mathbf{U}}^{\sharp}\right)^{(n)} \delta \mathbf{U}^{(n)} \\
& =-G^{\sharp}\left(\mathbf{U}_{\ell}, \mathbf{U}_{r}^{0}\right)+G^{\sharp}\left(\mathbf{U}_{\ell}, \mathbf{U}_{r}^{0}\right)-G^{\sharp}\left(\mathbf{U}_{\ell}, \mathbf{U}^{(n)}\right)+\left(G_{\mathbf{U}}^{\sharp}\right)^{(n)} \delta \mathbf{U}^{(n)} .
\end{aligned}
$$

We have

$$
\left\|G^{\sharp}\left(\mathbf{U}_{\ell}, \mathbf{U}_{r}^{0}\right)-G^{\sharp}\left(\mathbf{U}_{\ell}, \mathbf{U}^{(n)}\right)+\left(G^{\sharp}\right)^{(n)}(\delta \mathbf{U})^{(n)}\right\|_{C^{1, \alpha}\left(\Gamma_{i}\right)} \leq C\left\|\delta \mathbf{U}^{(n)}\right\|_{C^{1, \alpha}\left(\Gamma_{i}\right)}^{2} .
$$

Hence we obtain the estimate

$$
\left\|\left(g^{\sharp}\right)^{(n)}\right\|_{C^{1, \alpha}\left(\Gamma_{i}\right)} \leq C\left(\varepsilon+\left\|\delta \mathbf{U}^{(n)}\right\|_{C^{1, \alpha}\left(\Gamma_{i}\right)}^{2}\right) .
$$

Similarly,

$$
\left\|-H_{j}^{(n)}+H_{1 \mathbf{U}}^{(n)} \delta \mathbf{U}^{(n)}\right\|_{C^{0, \alpha}\left(\bar{\omega}_{i}\right)} \leq C\left(\varepsilon+\sum_{i}\left\|\delta \mathbf{U}^{(\mathbf{n})}\right\|_{C^{0, \alpha}\left(\bar{\omega}_{i}\right)}^{2}\right) .
$$

Therefore, by using Theorem 7.3 we obtain that the solution to problem $L^{(n)}$ exists and satisfies

$$
\sum_{i}\left\|\delta \mathbf{U}^{(n+1)}\right\|_{C^{1, \alpha}\left(\bar{\omega}_{i}\right)} \leq C\left(\varepsilon+\sum_{i}\left\|\delta \mathbf{U}^{(\mathbf{n})}\right\|_{C^{1, \alpha}\left(\bar{\omega}_{i}\right)}^{2}\right),
$$

where $C$ is independent of $\epsilon, \zeta, \delta$ and $n$.

From (8.4) we can easily prove the boundedness of $\delta \mathbf{U}^{(n)}$ in $C^{1, \alpha}\left(\bar{\omega}_{i}\right)$ by induction. Indeed, suppose that $\sum_{i}\left\|\delta \mathbf{U}^{(n)}\right\|_{C^{1, \alpha}\left(\bar{\omega}_{i}\right)} \leq \delta$ is valid for $n \leq n_{0}$. Then

$$
\sum_{i}\left\|\delta \mathbf{U}^{\left(n_{0}+1\right)}\right\|_{C^{1, \alpha}\left(\bar{\omega}_{i}\right)} \leq C\left(\varepsilon+\sum_{i}\left\|\delta \mathbf{U}^{\left(n_{0}\right)}\right\|_{C^{1, \alpha}\left(\bar{\omega}_{i}\right)}^{2}\right) \leq C\left(\varepsilon+\delta^{2}\right) .
$$

By taking $\delta \leq \frac{1}{2 C}$ and $\varepsilon \leq \frac{\delta}{2 C}$, we obtain

$$
\sum_{i}\left\|\delta \mathbf{U}^{\left(n_{0}+1\right)}\right\|_{C^{1, \alpha}\left(\bar{\omega}_{i}\right)} \leq \delta
$$

Hence the uniform boundedness of $\left\{\delta \mathbf{U}^{(n)}\right\}$ in $C^{1, \alpha}$ is proved.

Theorem 8.2. If the sequence $\left\{\delta \mathbf{U}^{(n+1)}\right\}$ of solutions of the problem $L^{(n)}$ is defined in Theorem 8.1, then $\left\{p^{(n)}, W^{(n)}\right\}$ is convergent in $C^{1, \alpha},\left\{U^{(n)}, \rho^{(n)}, \mu^{(n)}\right\}$ is convergent in $C^{0, \alpha}$. 
Proof. Let us derive a boundary value problem for $\Delta \mathbf{U}^{(n)}=\mathbf{U}^{(n+1)}-\mathbf{U}^{(n)}$. Obviously, $\Delta \mathbf{U}^{(n)}$ satisfies

(8.8)

$$
\left\{\begin{array}{c}
D_{R}^{(n)} \Delta W^{(n)}-e^{(n)} D_{I}^{(n)} \Delta p^{(n)}=\left(D_{R}^{(n-1)}-D_{R}^{(n)}\right) \delta W^{(n)}-\left(e^{(n-1)} D_{I}^{(n-1)}\right. \\
\left.-e^{(n)} D_{I}^{(n)}\right) \delta p^{(n)}-H_{1}^{(n)}+H_{1}^{(n-1)}+H_{1 \mathbf{U}}^{(n-1)}\left(\Delta \mathbf{U}^{(n-1)}\right), \\
D_{I}^{(n)} \Delta W^{(n)}+e^{(n)} D_{R}^{(n)} \Delta p^{(n)}=\left(D_{I}^{(n-1)}-D_{I}^{(n)}\right) \delta W^{(n)} \\
\quad+\left(e^{(n-1)} D_{R}^{(n-1)}-e^{(n)} D_{R}^{(n)}\right) \delta p^{(n)}-H_{2}^{(n)}+H_{2}^{(n-1)}+H_{2 \mathbf{U}}^{(n-1)}\left(\Delta \mathbf{U}^{(n-1)}\right), \\
\frac{\partial}{\partial x} \Delta U^{(n)}+d_{W}^{(n)} \frac{\partial}{\partial x} \Delta W^{(n)}+d_{p}^{(n)} \frac{\partial}{\partial x} \Delta p^{(n)} \\
\quad\left(d_{W}^{(n-1)}-d_{W}^{(n)}\right) \frac{\partial}{\partial x} \delta W^{(n)}+\left(d_{p}^{(n-1)}-d_{p}^{(n)}\right) \frac{\partial}{\partial x} \delta p^{(n)}, \\
\frac{\partial}{\partial x} \Delta p^{(n)}-\left(\frac{\gamma p}{\rho}\right)^{(n)} \frac{\partial}{\partial x} \Delta \rho^{(n)}=\left(\left(\frac{\gamma p}{\rho}\right)^{(n)}-\left(\frac{\gamma p}{\rho}\right)^{(n-1)}\right) \frac{\partial}{\partial x} \delta \rho^{(n+1)}, \\
U^{(n)} \frac{\partial}{\partial x} \Delta \mu^{(n)}-2 \Delta \mu^{(n)}=\left(U^{n-1)}-U^{(n)}\right) \frac{\partial}{\partial x} \Delta \mu^{(n)}, \\
\left(G_{\mathbf{U}}^{\sharp}\right)^{(n)}\left(\Delta \mathbf{U}^{(n)}\right)=-G^{\sharp}\left(\mathbf{U}_{\ell}, \mathbf{U}^{(n)}\right)+G^{\sharp}\left(\mathbf{U}_{\ell}, \mathbf{U}^{(n-1)}\right)+\left(G_{\mathbf{U}}^{\sharp}\right)^{(n-1)}\left(\Delta \mathbf{U}^{(n-1)}\right) \\
\Delta \mu^{(n)}=0 \text { on } \Gamma_{i} \quad(i=2,3), \quad(\sharp=a, b, c, \quad i=2,3), \\
\Delta p^{(n)}=0 \text { on } \Gamma_{L}, \quad \begin{array}{c}
(i) \\
\Delta p^{(n)}, \Delta W^{(n)} \quad \text { are continuous on } D^{T} .
\end{array}
\end{array}\right.
$$

As with the proof of Theorem 8.1, we have

$$
\begin{gathered}
\left\|-H_{j}^{(n)}+H_{j}^{(n-1)}+H_{j \mathbf{U}}^{(n-1)}\right\|_{C^{\alpha}\left(\bar{\omega}_{i}\right)} \leq C\left\|\Delta \mathbf{U}^{(n-1)}\right\|_{C^{\alpha}\left(\bar{\omega}_{i}\right)}^{2} \quad(j=1,2), \\
\|-G^{\sharp}\left(\mathbf{U}_{\ell}, \mathbf{U}^{(n)}\right)+ \\
G^{\sharp}\left(\mathbf{U}_{\ell}, \mathbf{U}^{(n-1)}\right)+\left(G_{\mathbf{U}}^{\sharp}\right)^{(n-1)}\left(\Delta \mathbf{U}^{(n-1)}\right) \|_{C^{1, \alpha}\left(\Gamma_{i}\right)} \\
\leq C\left\|\Delta \mathbf{U}^{(n-1)}\right\|_{C^{1, \alpha}\left(\Gamma_{i}\right)}^{2} .
\end{gathered}
$$

Besides, Theorem 8.1 indicates $\mathbf{U}^{(n)} \in \Sigma_{\delta}$. Therefore, by using Theorem 7.2, the first two equations in (8.8) yield

$$
\begin{aligned}
\left\|\Delta p^{(n)}, \Delta W^{(n)}\right\|_{C^{1, \alpha}\left(\bar{\omega}_{i}\right)} \leq C & \sum_{i=2,3}\left(\left\|\Delta \mathbf{U}^{(n-1)}\right\|_{C^{1, \alpha}\left(\Gamma_{i}\right)}^{2}\right. \\
& \left.+\left\|\Delta \mathbf{U}^{(n-1)}\right\|_{C^{\alpha}\left(\bar{\omega}_{i}\right)}^{2}+\delta\left\|\Delta \mathbf{U}^{(n-1)}\right\|_{C^{\alpha}\left(\bar{\omega}_{i}\right)}\right) .
\end{aligned}
$$

The three transport equations for $\Delta U^{(n)}, \Delta \rho^{(n)}, \Delta \mu^{(n)}$ indicate that their $C^{0, \alpha}$ norms are also dominated by the right hand side of (8.11). Hence define

$\left.M_{n, \alpha}=\left\|\Delta p^{(n)}, \Delta W^{(n)}\right\|_{C^{1, \alpha}\left(\bar{\omega}_{i}\right)}+\left\|\Delta U^{(n)}, \Delta \rho^{(n)}, \Delta \mu^{(n)}\right\|_{C^{0, \alpha}\left(\bar{\omega}_{i}\right)}+\| \Delta \mathbf{U}^{(n)}\right]\left.\right|_{C^{\alpha}\left(\Gamma_{i}\right)}$.

We have

$$
M_{n, \alpha} \leq \frac{1}{2} M_{n-1, \alpha}
$$

if $\delta$ is sufficiently small. Inequality (8.12) implies the convergence of $\left(W^{(n)}, p^{(n)}\right)$ in $C^{1, \alpha}$ and $\left(U^{(n)}, \rho^{(n)}, \mu^{(n)}\right)$ in $C^{0, \alpha}$.

The solvability of the nonlinear fixed boundary value problem (NL). Let $n \rightarrow \infty ;\left\{\mathbf{U}^{(n)}\right\}$ is convergent according to the above theorem. The limit $\mathbf{U} \in C^{\alpha}\left(\bar{\omega}_{i}\right)$, while $W$ and $p$ are also in $C^{1, \alpha}\left(\bar{\omega}_{i}\right)$. Moreover, since $\left\{\mathbf{U}^{(n)}\right\}$ is uniformly bounded in $C^{1, \alpha}(\bar{\omega})$, then the limit $\mathbf{U}$ also belongs to $C^{1, \alpha}\left(\bar{\omega}_{i}\right)$. It satisfies 
SHUXING CHEN

the system (5.10) in $\bar{\omega}_{i}$ and the boundary condition on $\Gamma_{i}$ and $\Gamma_{L}$. The function $p$ and $W$ also belong to $H^{1}(\omega)$, so that it must be continuous on $y=0$.

In view of the boundedness of $\mathbf{U}^{(n)}$ in $C^{1, \alpha}$ as stated in Theorem 8.1, we also know that $\mathbf{U}^{(n)}$ and $D \mathbf{U}^{(n)}$ are convergent at each point in $\omega_{2,3}$. Therefore, by taking the limit in (8.4), we obtain

$$
\sum_{i}\|\delta \mathbf{U}\|_{C^{1, \alpha}\left(\bar{\omega}_{i}\right)} \leq C\left(\epsilon+\|\delta \mathbf{U}\|_{C^{1, \alpha}\left(\bar{\omega}_{i}\right)}^{2}\right) \leq C\left(\epsilon+\delta \sum_{i}\|\delta \mathbf{U}\|_{C^{1, \alpha}\left(\bar{\omega}_{i}\right)}\right), \quad i=2,3,
$$

which implies the estimate

$$
\sum_{i}\|\delta \mathbf{U}\|_{C^{1, \alpha}\left(\bar{\omega}_{i}\right)} \leq 2 C \varepsilon, \quad i=2,3
$$

provided $\delta<\frac{1}{2 C}$.

\section{Proof of the MAIN THEOREM}

The solvability of the free boundary value problem.

Now we use the fixed point theorem to prove Theorem 2.1. As proved in Section 8 , for any $\left(\chi_{2}(x), \chi_{3}(x)\right) \in K_{\zeta}$ we have the solutions $\mathbf{U}_{2}(x, y)$ and $\mathbf{U}_{3}(x, y)$ to the problem $(N L)$. Then we define $\Xi_{i}(x)$ by

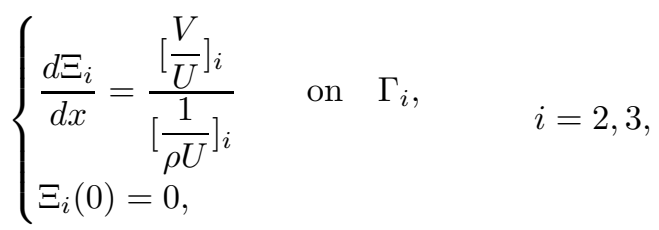

which gives a map

$$
\pi:\left(\chi_{2}(x), \chi_{3}(x)\right) \mapsto\left(\Xi_{2}(x), \Xi_{3}(x)\right) .
$$

Obviously, to prove the unique existence of the solution to the problem $(F B)_{1}$, we only need to prove that the map $\pi$ has a unique fixed point in $K_{\zeta}$.

As mentioned in Section 2, the value of $\mathbf{U}_{0}^{0}, \mathbf{U}_{1}^{0}, \psi_{1}^{0}$ and the boundary conditions in (NL) can completely determine the unperturbed flat Mach configuration. Then the values of $\mathbf{U}_{2}, \mathbf{U}_{3}$ at the origin are equal to $\mathbf{U}_{2}^{0}, \mathbf{U}_{3}^{0}$, so that (9.1) gives $\Xi_{i}^{\prime}(0)=\chi_{i}^{0}$ for $i=2,3$. Denote the right hand side of (9.1) by $J\left(\mathbf{U}_{\ell i}, \mathbf{U}_{i}\right)$. We write

$$
J\left(\mathbf{U}_{\ell i}, \mathbf{U}_{i}\right)=J\left(\mathbf{U}_{\ell i}, \mathbf{U}_{i}\right)-J\left(\mathbf{U}_{\ell i}^{0}, \mathbf{U}_{i}\right)+J\left(\mathbf{U}_{\ell i}^{0}, \mathbf{U}_{i}\right)-J\left(\mathbf{U}_{\ell i}^{0}, \mathbf{U}_{i}^{0}\right)+J\left(\mathbf{U}_{\ell i}^{0}, \mathbf{U}_{i}^{0}\right) .
$$

In view of $J\left(\mathbf{U}_{\ell i}^{0}, \mathbf{U}_{i}^{0}\right)=\chi_{i}^{0}$ and $\left\|\mathbf{U}_{i}-\mathbf{U}_{i}^{0}\right\|_{C^{1, \alpha}\left(\bar{\omega}_{i}\right)} \leq C \varepsilon$, we have

$$
\left\|\frac{d \Xi_{i}(x)}{d x}-\chi_{i}^{0}\right\|_{C^{1, \alpha}\left(\Gamma_{i}\right)}=\left\|J\left(\mathbf{U}_{\ell i}, \mathbf{U}_{i}\right)-\chi_{i}^{0}\right\|_{C^{1, \alpha}\left(\Gamma_{i}\right)} \leq C \varepsilon, \quad i=2,3 .
$$

Hence

$$
\left\|\Xi_{i}(x)-\chi_{i}^{0} x\right\|_{C^{2, \alpha}\left(\Gamma_{i}\right)} \leq C L_{0} \epsilon, \quad i=2,3 .
$$

Taking $\varepsilon, \zeta$ sufficiently small and letting $C L_{0} \varepsilon<\zeta$, the map $\pi$ from $\left(\chi_{2}, \chi_{3}\right)$ to $\left(\Xi_{2}, \Xi_{3}\right)$ is an inner map from $K_{\zeta}$ to $K_{\zeta}$.

Next we prove that the map $\pi$ is contractive in $C^{1, \alpha}$. Suppose that we have two couples $\left(\chi_{2 A}(x), \chi_{3 A}(x)\right)$ and $\left(\chi_{2 B}(x), \chi_{3 B}(x)\right)$ in $K_{\zeta}$. The solutions of $(N L)$ 
corresponding to these two couples of boundaries are $\mathbf{U}_{A}$ and $\mathbf{U}_{B}$, respectively. As with the transformation (7.1), we define

$$
\Pi_{A}: \tilde{x}=x, \quad \tilde{y}= \begin{cases}-\frac{y}{\chi_{2 A}(x)} x & \text { if } y<0, \\ \frac{y}{\chi_{3 A}(x)} x & \text { if } y>0 .\end{cases}
$$

Then the problem $(N L)$ is reduced to a corresponding problem on $\omega=\omega_{+} \cup \omega_{-}$. Still denote the solution under transformation $\pi_{A}$ by $\mathbf{U}_{A}$. It satisfies

$$
\left\{\begin{array}{l}
D_{R A} W_{A}-e_{A} D_{I A} p_{A}+\frac{\lambda_{R A}}{\rho_{A} U_{A}^{2}}=0 \text { in } \omega_{ \pm}, \\
D_{I A} W_{A}+e_{A} D_{R A} p_{A}+\frac{\lambda_{I A}}{\rho_{A} U_{A}^{2}}=0 \text { in } \omega_{ \pm}, \\
\frac{\partial U_{A}}{\partial x}+d_{W A} \frac{\partial W_{A}}{\partial x}+d_{p A} \frac{\partial p_{A}}{\partial x}+1=0 \text { in } \omega_{ \pm}, \\
\frac{\partial p_{A}}{\partial x}-\frac{\gamma p_{A}}{\rho_{A}} \frac{\partial \rho_{A}}{\partial x}=0 \text { in } \omega_{ \pm}, \\
U_{A} \frac{\partial \mu_{A}}{\partial x}-2 \mu_{A}=0 \text { in } \omega_{ \pm}, \\
G^{\sharp}\left(U_{\ell}, \mathbf{U}_{A}\right)=0 \text { on } \Gamma_{ \pm}(\sharp=a, b, c), \\
\mu_{A}=1 \text { on } \Gamma_{ \pm}, \\
W_{A}, p_{A} \text { are continuous on } \Gamma_{D}, \\
p_{A}=p_{0} \text { on } \Gamma_{L},
\end{array}\right.
$$

where the coefficients depend on $\mathbf{U}_{A}$ and the first derivatives of $\left(\chi_{2 A}(x), \chi_{3 A}(x)\right)$. Similarly, (9.5) also holds, if the subscript " $A$ " is replaced by " $B$ ".

Simply writing the five equations in (9.5) as $F_{\mathbf{U}_{A}}\left[\mathbf{U}_{A}\right]=0$ and letting $\mathbf{U}_{B A}=$ $\mathbf{U}_{B}-\mathbf{U}_{A}, \chi_{B A}=\chi_{B}-\chi_{A}$, we have

$$
\left\{\begin{array}{l}
F_{\mathbf{U}_{A}}\left[\mathbf{U}_{B A}\right]=F_{\mathbf{U}_{A}}\left[\mathbf{U}_{B}\right]-F_{\mathbf{U}_{B}}\left[\mathbf{U}_{B}\right]\left(\triangleq f_{B A}\right) \text { in } \omega_{ \pm}, \\
G_{\mathbf{U}}^{\sharp}\left(\mathbf{U}_{\ell}\left(x, \chi_{A}(x)\right), \mathbf{U}^{*}\right) \mathbf{U}_{B A}=g_{B A}^{\sharp} \text { on } \Gamma_{ \pm}(\sharp=a, b, c), \\
\mu_{B A}=0 \text { on } \Gamma_{ \pm}, \\
W_{B A}, p_{B A} \text { are continuous on } \Gamma_{D}, \\
p_{B A}=0 \text { on } \Gamma_{L},
\end{array}\right.
$$

where $g_{B A}^{\sharp}=G^{\sharp}\left(\mathbf{U}_{\ell}\left(x, \chi_{A}(x)\right), \mathbf{U}_{B}\right)-G^{\sharp}\left(\mathbf{U}_{\ell}\left(x, \chi_{B}(x)\right), \mathbf{U}_{B}\right)$ and $\mathbf{U}^{*}$ is a point in between $\mathbf{U}_{A}$ and $\mathbf{U}_{B}$. Since $\mathbf{U}_{A}$ and $\mathbf{U}_{B}$ satisfy (9.6), then for the right hand side of the equations in (9.6) we have estimates

$$
\begin{gathered}
\left\|f_{B A}\right\|_{C^{\alpha}\left(\bar{\omega}_{ \pm}\right)} \leq C \varepsilon\left(\left\|\mathbf{U}_{B A}\right\|_{C^{\alpha}\left(\bar{\omega}_{ \pm}\right)}+\left\|\chi_{B A}\right\|_{C^{1, \alpha}(0, \ell)}\right), \\
\left\|g_{B A}^{\sharp}\right\|_{C^{1, \alpha}\left(\Gamma_{ \pm}\right)} \leq C \varepsilon\left\|\chi_{B A}\right\|_{C^{1, \alpha}(0, \ell)} .
\end{gathered}
$$

Therefore, by using the result in the previous section, we obtain

$$
\left\|\mathbf{U}_{B A}\right\|_{C^{\alpha}\left(\bar{\omega}_{ \pm}\right)} \leq C \epsilon\left\|\chi_{B A}\right\|_{C^{1, \alpha}(0, \ell)} .
$$


Furthermore, by using the equation (9.1) for $\Xi_{i A}$ and $\Xi_{i B}$, we have

$$
\begin{gathered}
\left\|\Xi_{i B}(x)-\Xi_{i A}(x)\right\|_{C^{1, \alpha}(0, \ell)} \leq C\left\|\frac{d \Xi_{i B}}{d x}-\frac{d \Xi_{i A}}{d x}\right\|_{C^{\alpha}(0, \ell)} \\
\leq\left\|J\left(\mathbf{U}_{\ell}, \mathbf{U}_{B}\right)-J\left(\mathbf{U}_{\ell}, \mathbf{U}_{A}\right)\right\|_{C^{\alpha}(0, \ell)} \\
\leq C\left\|U_{B A}\right\|_{C^{\alpha}\left(\bar{\omega}_{ \pm}\right)} .
\end{gathered}
$$

Combining with (9.7), we obtain the contraction of the map $\pi$ in $C^{1, \alpha}$, provided $\varepsilon$ is small enough. This fact ensures that the map $\pi$ has a unique fixed point in $K_{\zeta}$. Hence the existence of the problem $(F B)_{1}$ is proved. Moreover, the estimates

$$
\begin{array}{r}
\|\mathbf{U}\|_{C^{1, \alpha}\left(\bar{\omega}_{i}\right)} \leq C \varepsilon, \quad i=2,3, \\
\left\|\chi_{i}(x)-\chi_{i}^{0} x\right\|_{C^{1, \alpha}(0, \ell)} \leq C \varepsilon, \quad i=2,3,
\end{array}
$$

can be obtained from (8.13) and (9.3) with different constants $C$.

The proof of Theorem 2.1. The problem $(F B)_{1}$ is deduced from the problem $(F B)$ in Section 3. By using the inverse transform of (3.2), we can obtain the solution of problem $(F B)$. Indeed, for the components of the solution $\mathbf{U}$ of $(F B)_{1}, \frac{V}{U} d x+$ $\frac{1}{\mu \rho U} d y$ is a total differential form due to (3.6). Therefore, the transform

$$
\xi=x, \quad \eta=\int_{(0,0)}^{(x, y)} \frac{V}{U} d x+\frac{1}{\mu \rho U} d y
$$

is well defined, which is the inverse of the transformation $T$ defined by (3.3). Therefore, it is easy to check that $T^{-1} \mathbf{U}$ satisfies the system (2.1). The images of $\Gamma_{2}$ and $\Gamma_{3}$ under the transform $T^{-1}$ are the shocks $S_{2}$ and $S_{3}$, while the image of $\Gamma_{D}$ is the slip line $\tilde{D}$.

The estimates (2.20) and (2.19) with $i=2,3$ can be obtained from (9.8), (9.9) with a transformation $T^{-1}$. Finally, $\|\mathbf{U}\|_{C^{1, \alpha}} \leq C \varepsilon$ implies

$$
\left\|\frac{V}{U}\right\|_{C^{1, \alpha}} \leq C \varepsilon
$$

with different $C$, because $U$ is bounded away from 0 . Correspondingly,

$$
\left\|\psi_{4}(\xi)\right\|_{C^{1, \alpha}(0, \ell)} \leq C\left\|\frac{d y}{d x}(x, 0)\right\|_{C^{0, \alpha}(0, \ell)} \leq C \varepsilon .
$$

Thus we obtain the unique existence of the solution described in Theorem 2.1. Correspondingly, Proposition 2.2 is then also valid. As we indicated in Section 2, Theorem 2.1 and Proposition 2.2 give the desired stability of Mach configuration.

\section{Appendix: Shock polar analysis and Assumption (A)}

In this appendix we are going to give some analysis of $(\theta, p)$ shock polar and then give the explanation of Assumption (A) in the main theorem. The notation applied in this Appendix may not be completely the same as before.

As mentioned in Section 2 the analysis of shock polar for stationary flow is also available for pseudo-stationary flow. Assume that the shock front moves with velocity $\xi_{0}$ and the flow parameters ahead of it and behind it are $\left(u_{0}, v_{0}, p_{1}, \rho_{0}\right)$ 
and $\left(u_{1}, v_{1}, p_{1}, \rho_{1}\right)$, respectively. If $u_{0}=v_{0}=v_{1}=0$, then from Rankine-Hugoniot conditions we have some basic relations as (see [14]):

$$
\begin{gathered}
\rho_{0}\left(\xi_{0}-u_{1}\right)=\rho_{0} \xi_{0}, \\
p_{1}+\rho_{1}\left(\xi_{0}-u_{1}\right)^{2}=p_{0}+\rho_{0} \xi_{0}^{2}, \\
\frac{1}{2}\left(u_{1}-\xi_{0}\right)^{2}+\frac{a_{1}^{2}}{\gamma-1}=\frac{1}{2} \xi_{0}^{2}+\frac{a_{0}^{2}}{\gamma-1} .
\end{gathered}
$$

For polytropic gas, $a^{2}=\frac{\gamma p}{\rho}$. Denoting $\mu^{2}=\frac{\gamma-1}{\gamma+1}$, one has

$$
\frac{\rho_{1}}{\rho_{0}}=\frac{p_{1}+\mu^{2} p_{0}}{p_{0}+\mu^{2} p_{1}}
$$

In the study of the pseudo-stationary flow described in Section 2 near $\left(\xi_{0}, \eta_{0}\right)$, we have $u_{0}=v_{0}=0, \eta_{0}=\xi_{0} \tan \theta_{*}$ and $(U, V)=\left(u-\xi_{0}, v-\eta_{0}\right)$. In this case the pseudo-Mach numbers are

$$
M_{0}=\frac{\left(\xi_{0}^{2}+\eta_{0}^{2}\right)^{1 / 2}}{\gamma p_{0} / \rho_{0}}, \quad M_{1}=\frac{\left(\left(\xi_{0}-u_{1}\right)^{2}+\eta_{0}^{2}\right)^{1 / 2}}{\gamma p_{1} / \rho_{1}} .
$$

$M_{1}$ can also be written as a function of $p_{1} / p_{0}$ and $M_{0}$. In fact, since a Mach number is independent of any rotation of the coordinate system, the last equation of (10.1) implies

$$
\frac{a_{1}^{2}}{a_{0}^{2}}\left(\frac{1}{2} M_{1}^{2}+\frac{1}{\gamma-1}\right)=\frac{1}{2} M_{0}^{2}+\frac{1}{\gamma-1} .
$$

Then by using (10.2), we obtain

$$
\frac{1}{2} M_{1}^{2}+\frac{1}{\gamma-1}=\left(\frac{1}{2} M_{0}^{2}+\frac{1}{\gamma-1}\right) \frac{\mu^{2}+\frac{p_{1}}{p_{0}}}{\frac{p_{1}}{p_{0}}+\mu^{2}\left(\frac{p_{1}}{p_{0}}\right)^{2}} .
$$

For given $p_{0}, M_{0}$, the $(\theta, p)$ shock polar is determined by the equation (see [14])

$$
\tan \theta=\frac{\frac{p}{p_{0}}-1}{\gamma M_{0}^{2}-\frac{p}{p_{0}}+1} \cdot \sqrt{\frac{\left(1+\mu^{2}\right)\left(M_{0}^{2}-1\right)-\left(\frac{p}{p_{0}}-1\right)}{\frac{p}{p_{0}}+\mu^{2}}} .
$$

The picture of the shock polar has been shown in Figure 2, which has $P_{0}$ as its double point. Any point on the shock polar gives a corresponding state, which can be connected by a shock front with the state $P_{0}$ (once $p$ is given, then other flow parameters can be determined by Rankine-Hugoniot conditions). When we are restricted to the state behind the shock front, the part with $p<p_{0}$ need not be considered, because the entropy condition indicates that the pressure $p$ behind a shock must be greater than the pressure $p_{0}$ ahead of the shock. Therefore, the effective shock polar on the $(\theta, p)$ plane forms a loop $\gamma_{0}$ with $P_{0}\left(0, p_{0}\right)$ as its double point, which will also be called a tip of the loop $\gamma_{0}$ later.

Besides, for shock polar $\gamma_{0}$ there is a number $\theta_{\max }$ such that the absolute value of the $\theta$-coordinate of any point on $\gamma_{0}$ is no more than $\theta_{\max }$. It means that the change of the direction of the pseudo-velocity across the shock front cannot be greater than $\theta_{\max }$. If $|\tilde{\theta}|<\theta_{\max }$, then there are two intersections of the straight line $\theta=\tilde{\theta}$ with the loop $\gamma_{0}$, corresponding to the weak shock and strong shock, respectively. 
For any point $P_{1}\left(\theta_{1}, p_{1}\right)$ on the lower part of the loop $\gamma_{0}$, the corresponding state is also pseudo-supersonic. Then, we can draw another shock polar $\gamma_{1}$ with $P_{1}$ as its double point. When $P_{1}$ runs along the loop $\gamma_{0}$, the corresponding parameter $M_{1}$ can be determined by using (10.5). Generally, the loops $\gamma_{0}$ and $\gamma_{1}$ will intersect at a point $P_{2}$ (or $P_{3}$ ) other than $P_{1}$. Indeed, we can prove that for suitably large $M_{0}$ and $P_{1}$, the height $h_{1}$ of the top point of $\gamma_{1}$ is larger than the height $h_{0}$ of the top point of $\gamma_{0}$. This fact means that the top point of $\gamma_{1}$ is outside of $\gamma_{0}$. Then $\gamma_{1}$ and $\gamma_{0}$ must intersect at a point $P_{2}$ different from $P_{1}$. To this end, we first prove the following lemma.

Lemma 10.1. If $M_{0}$ is suitably large and $P_{1}$ is near $P_{0}$, then

$$
\frac{d}{d p_{1}}\left(p_{\max }\right)_{1}>0
$$

Proof. Denote the right hand side of the equation (10.6) by $F\left(p, p_{0}, M_{0}\right)$. Then the equation of the curve $\gamma_{0}$ is

$$
\tan \theta=F\left(p, p_{0}, M_{0}\right)
$$

while the equation of the curve $\gamma_{1}$ is

$$
\tan \theta=F\left(p, p_{1}, M_{1}\right),
$$

where $M_{1}=M\left(p_{1}\right)$ is determined by (10.5). The highest point of $\gamma_{0}$ corresponds to the larger value of $p$ for $\theta=0$. From the equation (10.6) the coordinate $p$ at the highest point satisfies

$$
\left(1+\mu^{2}\right)\left(M_{0}^{2}-1\right)=\frac{p}{p_{0}}-1
$$

Hence

$$
\left(p_{\max }\right)_{0}=p_{0}\left(1+\mu^{2}\right)\left(M_{0}^{2}-1\right)+p_{0}
$$

Similarly, the $p$-coordinate of the top point of $\gamma_{1}$ is

$$
\left(p_{\max }\right)_{1}=p_{1}\left(1+\mu^{2}\right)\left(M\left(p_{1}\right)^{2}-1\right)+p_{1} .
$$

Therefore,

$$
\frac{d}{d p_{1}}\left(p_{\max }\right)_{1}=\left(1+\mu^{2}\right)\left(M\left(p_{1}\right)^{2}-1\right)+\left(1+\mu^{2}\right) p_{1} \frac{d M^{2}\left(p_{1}\right)}{d p_{1}}+1
$$

Denote $\frac{p_{1}}{p_{0}}$ by $\zeta$. We find from (10.5)

$$
\frac{1}{2} M^{2}\left(p_{1}\right)+\frac{1}{\gamma-1}=\left(\frac{1}{2} M_{0}^{2}+\frac{1}{\gamma-1}\right) \cdot \frac{\zeta+\mu^{2}}{\zeta+\mu^{2} \zeta^{2}} .
$$

Then

$$
\begin{gathered}
p_{1} \frac{d M^{2}\left(p_{1}\right)}{d p_{1}}=\left(\frac{1}{2} M_{0}^{2}+\frac{1}{\gamma-1}\right) \cdot\left(\frac{\zeta+\mu^{2}}{\zeta+\mu^{2} \zeta^{2}}\right)_{\zeta}^{\prime} \cdot \frac{p_{1}}{p_{0}} \\
=\left(\frac{1}{2} M_{0}^{2}+\frac{1}{\gamma-1}\right) \cdot \frac{-\mu^{2}\left(1+\zeta^{2}+2 \mu^{2} \zeta\right)}{\left(\zeta+\mu^{2} \zeta^{2}\right)^{2}} \zeta
\end{gathered}
$$


Substituting it into (10.11) and using (10.5) yield

$$
\begin{aligned}
& \frac{d}{d p_{1}}\left(p_{\max }\right)_{1}=\left(1+\mu^{2}\right) M_{1}^{2}+\mu^{2}+\left(1+\mu^{2}\right)\left(\frac{1}{2} M_{0}^{2}+\frac{1}{\gamma-1}\right) \frac{-\mu^{2}\left(1+\zeta^{2}+2 \mu^{2} \zeta\right)}{\left(\zeta+\mu^{2} \zeta^{2}\right)\left(1+\mu^{2} \zeta\right)} \\
& =\left(1+\mu^{2}\right)\left(\frac{1}{2} M_{0}^{2}+\frac{1}{\gamma-1}\right) \frac{2\left(\zeta+\mu^{2}\right)\left(1+\mu^{2} \zeta\right)-\mu^{2}\left(1+\zeta^{2}+2 \mu^{2} \zeta\right)}{\left(\zeta+\mu^{2} \zeta^{2}\right)\left(1+\mu^{2} \zeta\right)} \\
& \quad-\frac{2\left(1+\mu^{2}\right)}{\gamma-1}+\mu^{2} \\
& =\left(\frac{1}{2} M_{0}^{2}+\frac{1}{\gamma-1}\right) \frac{2 \zeta+\mu^{2}+\mu^{2} \zeta^{2}}{\zeta\left(1+\mu^{2} \zeta\right)^{2}}-\frac{1-2 \mu^{4}}{\mu^{2}} .
\end{aligned}
$$

Since $\left(\frac{2 \zeta+\mu^{2}+\mu^{2} \zeta^{2}}{\zeta\left(1+\mu^{2} \zeta\right)^{2}}\right)^{\prime}>0$ as $\zeta>1$, then $\frac{2 \zeta+\mu^{2}+\mu^{2} \zeta^{2}}{\zeta\left(1+\mu^{2} \zeta\right)^{2}}$ is monotonically increasing. Therefore, for any fixed $\zeta_{*}>1$, we can find $M_{0 *}$ depending on $\mu, \zeta_{*}$, such that $\frac{d}{d p_{1}}\left(p_{\max }\right)_{1}>0$ for $M>M_{0 *}$ and all $1<\zeta<\zeta_{*}$.

Next we prove that for $P_{1}$ on $\gamma_{0}$, the $\theta$-coordinate of the intersection $P_{2}$ is between $\theta\left(P_{0}\right)$ and $\theta\left(P_{1}\right)$.

Lemma 10.2. Denote by $w_{1}$ the width of the loop $\gamma_{1}$. We have $\frac{d w_{1}}{d p_{1}}<0$.

Proof. If the $\theta$-coordinate of the double point of $\gamma_{0}$ is zero, then the width $w$ of $\gamma_{0}$ equals $2 \theta_{\max }$, where $\theta_{\max }$ is the maximum of the value of the $\theta$-coordinate of the point on $\gamma_{0}$. From the equation (10.5) we know that if $p_{*}$ satisfies $F_{p}\left(p_{*}, p_{0}, M_{0}\right)=$ 0 , then the value of $|\theta|$ corresponding to $P_{*}$ is $\theta_{\max }$, i.e. $\tan \frac{w}{2}=F\left(p_{*}, p_{0}, M_{0}\right)$. Similarly, $\tan \frac{w_{1}}{2}=F\left(\left(p_{*}\right)_{1}, p_{1}, M_{1}\right)$, where $\left(p_{*}\right)_{1}$ satisfies $F_{p}\left(\left(p_{*}\right)_{1}, p_{1}, M_{1}\right)=0$. Therefore, we only need to show $\frac{d}{d p_{1}} F\left(\left(p_{*}\right)_{1}, p_{1}, M_{1}\right)<0$. By the chain rule

$$
\frac{d}{d p_{1}} F\left(\left(p_{*}\right)_{1}, p_{1}, M_{1}\right)=\frac{\partial F}{\partial p} \frac{\partial p_{*}}{\partial p_{1}}+\frac{\partial F}{\partial p_{1}}+\frac{\partial F}{\partial\left(M_{1}^{2}\right)} \frac{d\left(M_{1}^{2}\right)}{d p_{1}} .
$$

According to the meaning of $p_{*}$, the first term on the right hand side of the equation is zero. By using the expression of $F\left(p, p_{0}, M_{0}\right)$, we know $F_{p}$ takes the form $f\left(\frac{p}{p_{0}}, M_{0}\right) \frac{1}{p_{0}}$, and $F_{p_{0}}$ takes the form $f\left(\frac{p}{p_{0}}, M_{0}\right)\left(-\frac{p}{p_{0}^{2}}\right)$. Hence $\frac{\partial F}{\partial p}\left(\left(p_{*}\right)_{1}, p_{1}, M_{1}\right)=$ 0 also implies $\frac{\partial F}{\partial p_{1}}\left(\left(p_{*}\right)_{1}, p_{1}, M_{1}\right)=0$. It turns out that the sign of the right hand side of (10.14) is determined by its last term in the right hand side. For $\zeta=\zeta_{*}$

$$
\begin{aligned}
& \frac{\partial F}{\partial\left(M_{1}^{2}\right)}=\frac{-\gamma\left(\zeta_{*}-1\right)}{\left(\gamma M_{1}^{2}-\left(\zeta_{*}-1\right)\right)^{2}}(Q)^{\frac{1}{2}}+\frac{\zeta_{*}-1}{\gamma M_{1}^{2}-\left(\zeta_{*}-1\right)} \cdot \frac{1}{2}(Q)^{-\frac{1}{2}} \frac{1+\mu^{2}}{\zeta_{*}+\mu^{2}} \\
& \quad=\frac{\zeta_{*}-1}{\left(\gamma M_{1}^{2}-\left(\zeta_{*}-1\right)\right)^{2}}(Q)^{-\frac{1}{2}} \\
& \quad \cdot\left[\frac{-\gamma\left(\left(1+\mu^{2}\right)\left(M_{1}^{2}-1\right)-\left(\zeta_{*}-1\right)\right)}{\zeta_{*}+\mu^{2}}+\frac{\frac{1}{2}\left(1+\mu^{2}\right)\left(\gamma M_{1}^{2}-\left(\zeta_{*}-1\right)\right)}{\zeta_{*}+\mu^{2}}\right],
\end{aligned}
$$


where $(Q)=\frac{\left(1+\mu^{2}\right)\left(M_{1}^{2}-1\right)-\left(\zeta_{*}-1\right)}{\zeta_{*}+\mu^{2}}$. In view of $f_{\zeta}=0$ at $\zeta=\zeta_{*}$, we have

$$
\frac{\gamma M_{1}^{2}}{\gamma M_{1}^{2}-\zeta_{*}+1} \cdot \frac{\left(1+\mu^{2}\right)\left(M_{1}^{2}-1\right)-\left(\zeta_{*}-1\right)}{\zeta^{*}+\mu^{2}}+\frac{\zeta_{*}-1}{2} \cdot \frac{-\left(1+\mu^{2}\right) M_{1}^{2}}{\left(\zeta_{*}+\mu^{2}\right)^{2}}=0,
$$

or

$$
2 \gamma\left[\left(1+\mu^{2}\right)\left(M_{1}^{2}-1\right)-\left(\zeta_{*}-1\right)\right]=\left(1+\mu^{2}\right)\left(\zeta_{*}-1\right)\left(\gamma M_{1}^{2}-\zeta+1\right) /\left(\zeta_{*}+\mu^{2}\right) .
$$

Substituting it into (10.15), direct computation then indicates

$$
\frac{\partial F}{\partial\left(M_{1}^{2}\right)}=\frac{\zeta_{*}-1}{\gamma M_{1}^{2}-\zeta_{*}+1}(Q)^{-\frac{1}{2}} \frac{\left(1+\mu^{2}\right)^{2}}{\left(\zeta_{*}+\mu^{2}\right)^{2}}>0 .
$$

On the other hand, from (10.13) we know $\frac{d M^{2}\left(p_{1}\right)}{d p_{1}}<0$. Hence (10.14) gives $\frac{d F}{d p_{1}}<0$, and the lemma is thus proved.

Choose the $(\theta, p)$ coordinate system to let $\theta\left(P_{0}\right)=0$. Lemma 10.2 claims that $w_{1}$ is monotonically decreasing, when $\theta\left(P_{1}\right)$ runs from 0 to $\frac{w_{0}}{2}$. So there is a critical number $\theta_{\alpha} \quad\left(<\theta_{\max }\right)$, such that

$$
\theta\left(P_{1}\right) \in\left(\theta_{\alpha}, \theta_{\max }\right) \quad \text { implies } \frac{w_{1}}{2}<\theta\left(P_{1}\right) .
$$

Therefore, we have

$$
0<\theta\left(P_{1}\right)-\frac{w_{1}}{2}<\theta\left(P_{2}\right)<\theta\left(P_{1}\right), \text { if } \theta\left(P_{1}\right)>\theta_{\alpha} .
$$

Then the points $P_{0}, P_{1}, P_{2}, P_{3}$ will give us a flat Mach configuration as shown in Section 2.

Summing up, we know that the following assumptions are sufficient to ensure the realization of the picture as shown in Figure 2.

1. $\xi_{0}>a_{0}$. This means that the relative velocity ahead of the shock front is supersonic.

2. $\left(\xi_{0} \tan \theta_{*}\right)^{2}+\left(\xi_{0}-u_{1}\right)^{2}<a_{1}^{2}$. This condition means that the regular reflection at $\left(\xi_{0}, \xi_{0} \tan \theta_{*}\right)$ is impossible.

3. $M_{0}$ is suitably large, so that Lemma 10.1 holds.

4. $\theta_{P_{1}}>\theta_{\alpha}$, where $\theta_{\alpha}$ is the critical number defined in (10.17).

These four assumptions altogether are called Assumption (A), which is applied in the main theorem.

\section{ACKNOWLEDGMENTS}

Work on this paper is partially supported by the National Natural Science Foundation of China 10531020, the National Basic Research Program of China 2006CB805902 and the Doctorial Foundation of National Educational Ministry 20050246001.

\section{REFERENCES}

[1] S. Agmom, A. Douglis and L. Nirenberg, Estimates near the boundary for solutions of elliptic partial differential equations satisfying general boundary conditions I, Comm. Pure Appl. Math. 12 (1959), 623-777. MR0125307 (23:A2610) 
[2] S. Agmom, A. Douglis and L. Nirenberg, Estimates near the boundary for solutions of elliptic partial differential equations satisfying general boundary conditions II, Comm. Pure Appl. Math. 17 (1964), 35-92. MR0162050(28:5252)

[3] G. Ben-Dor, Shock Waves Reflection Phenomena, Springer-Verlag, New York, 1992.

[4] M. Brio and J. K. Hunter, Mach reflection for the two-dimensional Burgers equation, Phys. D 60 (1992), 148-207. MR1195600 (93i:35119)

[5] M. Costabel and M. Dauge, Construction of corner singularities for Agmon-DouglisNirenberg elliptic systems, Math. Nachr. 162 (1993), 209-237. MR.1239587 (94k:35090)

[6] M. Costabel and M. Dauge, Stable asymptotics for elliptic systems on plane domains with corners, Comm. P.D.E. 19 (1991), 1677-1766. MR.1294475 (95g:35051)

[7] S. Canic and B. Kerfitz, Riemann problems for the two-dimensional unsteady transonic small disturbance equation, SIAM J. Appl. Math. 58 (1998), 636-665. MR 1617618 (99f:35127)

[8] S. Canic, B. Kerfitz and G. Lieberman, A proof of existence of perturbed steady transonic shocks via a free boundary problem, Comm. Pure Appl. Math. 53 (2000), 484-511. MR:1733695 (2001m:76056)

[9] S. Canic, B. Kerfitz and E. H. Kim, A free boundary problems for unsteady transonic small disturbance equation: transonic regular reflection, Methods and Appl. Anal. 7(2000), 313336. MR:1869288 (2002h:76077)

[10] S. Canic, B. Kerfitz and E. H. Kim, A free boundary problems for a quasilinear degenerate elliptic equation: transonic regular reflection, Comm. Pure Appl. Math. 55 (2002), 71-92. MR1857880 (2003a:35206)

[11] Shuxing Chen, Linear approximation of shock reffection at a wedge with large angle, Comm. P.D.E. 21 (1996), 1103-1114. MR.1399192 (97f:35136)

[12] Shuxing Chen, Stability of Transonic Shock Front in M-D Euler System, Trans. Amer. Math. Soc. 307 (2005), 287-308. MR.2098096 (2005h:35278)

[13] Shuxing Chen, Stability of Mach Configuration, Comm. Pure Appl. Math. 59 (2006), 1-35. MR2180082 (2006i:35294)

[14] R. Courant and K. O. Friedrichs, Supersonic Flow and Shock Waves, Interscience Publishers Inc., New York, 1948. MR0029615 (10:637c)

[15] C. M. Dafermos, Hyperbolic Conservation Laws in Continuum Physics, Springer-Verlag, Berlin, Heiderberg, New York, 2000. MR1763936 (2001m:35212)

[16] M. Dauge, Elliptic Boundary Value Problems in Corner Domains - Smoothness and Asymptotics of Solutions, Lecture Notes in Mathematics, 1341, Springer-Verlag, Berlin, 1988. MR.961439 (91a:35078)

[17] P. Grisvard, Elliptic problems in nonsmooth domains, Monographs and Studies in Mathematics 24, Pitman, London 1987. MR775683 (86m:35044)

[18] D. Gilbarg and N. S. Trudinger, Elliptic Partial Differential Equations of Second Order, Second Edition, Grundlehren der Mathematischen Wissenschaften, 224, Springer, Berlin, New York, 1983. MR737190 (86c:35035)

[19] J. K. Hunter and M. Brio, Weak shock reflection, J. Fluid Mech. 410 (2000), 235-261. MR1761754 (2001a:76094)

[20] L. F. Henderson, Region and Boundaries for Diffracting Shock Wave Systems, Zeitshrift für Angewandte Mathematik und Mechanik 67 (1987), 73-88.

[21] Y. Li and M. Vogelius, Gradient estimates for solutions to divergence form elliptic equations with discontinuous coefficients, Arch. Rat. Mech. Anal. 153 (2000), 91-151. MR1770682 (2001m:35083)

[22] T. T. Li and W. C. Yu, Boundary value problem for quasi-linear hyperbolic systems, Duke Univ. Math. Ser. 5 (1985). MR0823237 (88g:35115)

[23] A. Majda, One perspective on open problems in multi-dimensional conservation laws, IMA Math. Appl. 29(1991), 217-237. MR1087085 (91m:35142)

[24] C. S. Morawetz, Potential theory for regular and Mach reflection of a shock at a wedge, Comm. Pure Appl. Math. 47(1994), 593-624. MR.1278346 (95g:76030)

[25] John von Neumann, Oblique Reflection of Shocks, U.S. Dept. Comm. Off. of Tech. Serv., Washigton, D.C., PB-37079 (1943).

[26] D. Serre, Ecoulements de fluides parfaits en deux variables indépendantes de type espace. Réflexion d'un choc plan par un dièdre compressif, Arch. Rational Mech. Anal. 132 (1995), 15-36. MR1360078 (96i:76106) 
[27] D. Serre, Shock reflection in gas dynamics, Handbook of Fluid Dynamics, 4, Elsevier, NorthHolland, 2005.

[28] J. Smoller, Shock waves and reaction-diffusion equations, Springer-Verlag, New York, 1983. MR688146(84d:35002)

School of Mathematical Sciences, Fudan University, and Key laboratory of Mathematics for Nonlinear Sciences (Fudan University, Ministry of Education), Shanghai 200433, People's Republic of China

E-mail address: sxchen@public8.sta.net.cn 